\title{
Resonances in the three-neutron system
}

\author{
H. Witała*, W. Glöckle \\ Institut für theoretische Physik II, Ruhr-Universität Bochum, D-44780 Bochum, Germany \\ * Institute of Physics, Jagellonian University, PL- 30059 Cracow, Poland
}

(April 17, 2021)

\begin{abstract}
A study of 3-body resonances has been performed in the framework of configuration space Faddeev equations. The importance of keeping a sufficient number of terms in the asymptotic expansion of the resonance wave function is pointed out. We investigated three neutrons interacting in selected force components taken from realistic nn forces. Three-neutron resonance pole trajectories connected to artificially enhanced nn forces could be found. The final pole positions corresponding to the actual force strengths could not be found due to the onset of numerical instabilities. The numerically reliable results, however, achieved makes it likely, that three-neutron resonance energies will have large imaginary parts and therefore physically not interesting. A straithforward application of complex scaling to 3-nucleon systems with realistic forces could not be controlled numerically.
\end{abstract}

PACS numbers: 21.30.+y, 21.45.+v, 24.10.-i, 25.10.+s 


\section{INTRODUCTION}

Three-nucleon $(3 \mathrm{~N})$ resonances have not yet been firmly established. The situation up to 1987 has been collected in [1]. A recent investigation [2] of the process ${ }^{3} \mathrm{He}\left(\pi^{-}, \pi^{+}\right) 3 n$ found no evidence of a resonance state of three neutrons. Earlier investigations [3], [4] pointed to resonances in the three neutron (3n) and three proton (3p) systems. The double charge exchange process on ${ }^{3} \mathrm{He}$ was also investigated in [5] criticizing previous work but pointing again to a $3 \mathrm{n}$ resonance, now around $(20-i 20) \mathrm{MeV}$. For the nnp system an excited state around $7 \mathrm{MeV}$ with a width of $\Gamma=0.6 \pm 0.3 \mathrm{MeV}$ has been suggested in [6]. A variational study based on complex scaling and simplified nucleon-nucleon (NN) forces was carried through in [7] with the prediction of a $3 n$ resonance at $E=(14-i 13) \mathrm{MeV}$ for a $J^{\pi}=3 / 2^{+}$state. Earlier theoretical studies [8], [9] might be useful in various aspects, but are not conclusive with respect to the actual position of $3 \mathrm{n}$ resonances. A virtual state of ${ }^{3} H$ with the quantum numbers of the ground state has been investigated in [10] by analytically continuing the momentum space Faddeev equation and even estimating three-nucleon force (3NF) effects. In the framework of the hyperspherical harmonic method and using complex scaling with model potentials a subthreshold $3 \mathrm{n}$ resonance for $J^{\pi}=1 / 2^{-}$has been located in [11]. Recently mathematical foundations have been laid on the analytical continuation of the three-body Faddeev equations into unphysical energy sheets [12] and an application thereof to the ${ }^{4} \mathrm{He}$ trimer appeared in 13 .

It is a theoretical challenge to determine the locations of the lowest lying $3 \mathrm{~N}$ resonances on unphysical sheets based on the most modern nuclear forces. Those forces are very successful in describing 3N scattering observables [14] which leads to the expectation that the corresponding 3N Hamiltonian will also locate $3 \mathrm{~N}$ resonances with a high degree of reliability. Like for the 3N bound state energy, where some theoretical underbinding results and $3 \mathrm{NF}^{\prime}$ 's are used to bridge that gap to the data point, also $3 \mathrm{~N}$ resonance positions might finally be fine-tuned in this manner. These future rigorous results would also be an important guidance for experiments and would clarify the hitherto controversial situation. 
For the $3 \mathrm{~N}$ system the bound state has been studied extensively in the last decades resulting in numerically precise and converged solutions of the 3N Faddeev equations [15], [16], [17], [18], [19]. Energy eigenvalues and wave functions have been generated with most modern nuclear Hamiltonians in momentum and coordinate space. Also numerically exact solutions of $3 \mathrm{~N}$ Faddeev equations for continuum states with the same Hamiltonians are now available in momentum space for a wide range of energies [14]. The first model solutions also in coordinate space appeared [20] and variational approaches matured and provide equally accurate solutions [21].

$3 \mathrm{~N}$ resonances, however, have not yet been investigated in a comparable rigorous manner and based on realistic NN forces to the best of our knowledge. In this article we would like to take first steps into such a direction.

In the two-nucleon $(2 \mathrm{~N})$ system the exact form of the wave function in configuration space is known outside the range of the potential. In the $3 \mathrm{~N}$ system only asymptotic expansions are known [22], [23] and methods how to apply them with sufficient accuracy, for instance in Faddeev equations, have still to be elaborated in that framework. There are first very promising results for $3 \mathrm{~N}$ scattering above the $3 \mathrm{~N}$ breakup threshold [20] though up to now only for simple S-wave model forces. To the best of our knowledge $3 \mathrm{~N}$ resonance wave functions, which asymptotically oscillate and grow exponentially, have not yet been determined as solutions of Faddeev equations and realistic forces. First attempts in this direction have been done in [24], however with very simple forces.

In this study we investigate 3-body resonances as solutions of configuration space Faddeev equations. The largest obstacle to find the resonance positions with sufficient precision is the approximate form of the resonance boundary condition. Ab initio it is not known at which coordinate values this form is valid with sufficient accuracy. For resonances this problem can be overcome in principle using the complex scaling method (CSM) [25], 26]. Thereby all relative coordinates are "rotated" into the complex plane leading to bound-state like, exponentially decreasing resonance wave function. This "rotation" of the coordinates into the complex plane works well for problems in atomic physics with potentials of inverse power 
laws. It will be shown in this article that in nuclear physics a straighforward application of CSM in the configuration space Faddeev form leads to serious numerical problems when the usual realistic NN interactions of Yukawa type and hard core behavior are used. Whether feasible modifications thereof can be found remains to be seen. Right now our experience is such, that the resonance energies can be gained with sufficient precision only if one does not leave the real r-axis and if one guaranties the exact boundary condition as close as possible by using a sufficient number of terms in its asymptotic form.

In section II we explain in detail the problems encountered when one applies the CSM to the nuclear resonances using the $2 \mathrm{~N}$ system as a play ground. The exact knowledge of the boundary conditions for this system allows one to check assumptions which can later be applied to $3 \mathrm{~N}$ resonances. The $3 \mathrm{~N}$ equations and the method for their solution are presented in section III. There we discuss results for 3 bosons interacting with simple, S-wave forces and more importantly for 3n's interacting via realistic neutron-neutron (nn) interactions. A summary is given in section IV. Information on the technical performance can be found in the Appendices A and B.

\section{RESONANCES IN THE 2N SYSTEM}

Searching resonances in a system of two-nucleons is a well defined problem and numerically well under control [27]. In spite of that we discuss it here and present some results due to its pedagogical simplicity, which enables us to present and test approaches which might be useful for finding resonances for three or more nucleons. In addition we can introduce our notation.

The $2 \mathrm{~N}$ resonances are associated with the poles of the S-matrix which are embedded in the fourth-quadrant of the complex k-plane $(\operatorname{Re}(\mathrm{k})>0, \operatorname{Im}(\mathrm{k})<0)$. They are solutions of the time-independent Schrödinger equation without incoming wave and the outgoing wave increasing exponentially at infinity. We introduce the coordinate space partial wave basis $\left|r, \alpha_{2}>\equiv\right| r,(l s) j t>$ where 


$$
<\vec{r}^{\prime} \mid r l m>=\frac{\delta\left(r^{\prime}-r\right)}{r r^{\prime}} Y_{l m}\left(\hat{r}^{\prime}\right)
$$

and where the orbital angular momentum 1 and total spin s are coupled to the total angular momentum $\mathrm{j}$. The isospin of the two nucleons is denoted by t. Then the resonance wave function $\psi(\vec{r})$ to a given $\mathrm{j}$ and $\mathrm{t}$ has the form

$$
\psi(\vec{r})=\sum_{l} \frac{\phi_{\alpha_{2}}(r)}{r}<\hat{r} \mid \alpha_{2}>
$$

It is written in terms of the reduced wave functions $\phi_{\alpha_{2}}(r)$ which fulfill the radial Schrödinger equation

$$
\sum_{\alpha_{2}^{\prime}}\left[\delta_{\alpha_{2} \alpha_{2}^{\prime}}\left(\frac{d^{2}}{d r^{2}}-\frac{l_{\alpha_{2}}\left(l_{\alpha_{2}}+1\right)}{r^{2}}\right)-\frac{m}{\hbar^{2}} V_{\alpha_{2} \alpha_{2}^{\prime}}(r)\right] \phi_{\alpha_{2}^{\prime}}(r)=-k^{2} \phi_{\alpha_{2}}(r) .
$$

The resonance conditions are such that $\psi$ has to be regular at the origin and purely outgoing. For the reduced amplitudes this amounts to

$$
\phi_{\alpha_{2}}(0)=0
$$

and

$$
\phi_{\alpha_{2}}(r) \propto r h_{l_{\alpha_{2}}}^{(1)}(k r)
$$

outside the range of the interaction. Here $h_{l}^{(1)}(k r)$ is the spherical Hankel function. As we shall demonstrate below it is mandatory to either use the exact form of the boundary condition (5) or to take into account at least the leading orders to find the resonances in the $2 \mathrm{~N}$ system with sufficient accuracy. In Eq.(3) $\mathrm{m}$ is the nucleon mass and $k^{2} \equiv \frac{m}{\hbar^{2}} E$. The complex energy E is embedded in the lower half plane of the second energy sheet. The boundary conditions (田) and (5) apply also to bound states with real, negative eigenvalues E. The matrix elements of the NN interaction are defined in terms of $V_{\alpha_{2} \alpha_{2}^{\prime}}(r)$ by

$$
<r \alpha_{2}|V| r^{\prime} \alpha_{2}^{\prime}>=\frac{\delta\left(r-r^{\prime}\right)}{r r^{\prime}} V_{\alpha_{2} \alpha_{2}^{\prime}}(r)
$$

A method of solving Eq. (3) is displayed in [28]. One chooses suitable grid points in $r$ and interpolates $\phi_{\alpha_{2}}(r)$ in terms of cubic splines. The boundary conditions (4) and (5) can 
be conveniently incorporated either by the absence of certain expansion coefficients in the spline expansion or by suitable relations among them. Then choosing appropriate collocation points in $r$ one ends up with a homogeneous algebraic set of equations. They can be handled by standard methods [28].

In systems with more than 2 nucleons the boundary conditions are known only via an asymptotic expansion and one has therefore to work with approximate boundary conditions. The knowledge of the exact resonance solutions in the $2 \mathrm{~N}$ system allow now to study the question pivotal for 3N systems: how precisely does one need to know the boundary conditions in order to get the resonance energies with sufficient accuracy? To study this problem one can use the series representation for the spherical Hankel functions

$$
h_{l}^{(1)}(x)=\sum_{m=0}^{l} \frac{(l+m) !}{m !(l-m) !} \frac{1}{2^{m}} \frac{\exp \left(i\left\{x-\frac{l-m+1}{2} \pi\right\}\right)}{x^{m+1}}
$$

and write the resonance wave function outside the interaction region in a form which resembles the asymptotic boundary condition in the 3N system (see Section III, Eq.(21))

$$
\phi(r)=\exp (i k r) \sum_{m=0}^{l} \frac{a_{m}}{(k r)^{m}}
$$

with $a_{m}$ being unknown constants independent from $\mathrm{k}$ and $\mathrm{r}$. Here, contrary to the $3 \mathrm{~N}$ system, the number of terms in the series is restricted by the orbital angular momenta contributing to the given total angular momentum $\mathrm{j}$ of a resonance.

In Table [ [ we present $2 \mathrm{~N}$ resonance positions in different $\mathrm{NN}$ states where the exact boundary condition have been imposed at different matching radii outside the interaction region. The NN interaction Reid93 [29] has been used and has been multiplied with a factor $\lambda_{V}$ (shown in column 2 of Table $\mathbb{Q}$ ). Using the exact boundary condition the resonance positions do not depend of course on the value of the matching radius. However, when the most crudely approximated boundary condition is applied, keeping in Eq. (8) only the term with $m=0$, the approximate resonance positions vary with the matching radius and up to

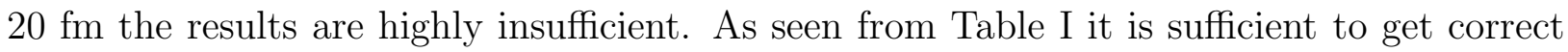
energy eigenvalues if one keeps only the two leading terms in the boundary condition (8) ( for $l=1$ this is the exact form). 
For resonances the problem of a possibly approximate nature of the boundary condition can be overcome in principle using the complex scaling method (CSM) [25], [26]. This will now be illustrated for 2 nucleons because of its simplicity. In the $2 \mathrm{~N}$ system one replaces the relative coordinate $r$ by

$$
r \rightarrow r^{\prime} \equiv r e^{i \alpha}
$$

For real positive angles $\alpha$ the asymptotic form $e^{i k \rho}$ with $\operatorname{Im}(k)<0$ can be changed to an exponentially decreasing function. Therefore a resonance wave function analytically continued to those complex coordinates can be treated like a bound state with its well known boundary condition. Then it is probably enough to restrict the boundary condition (8) to the first term $m=0$. This "rotation" of the coordinate into the complex plane is sufficient for problems in atomic physics with Coulomb potentials. In nuclear physics the potentials decrease exponentially and the transformation (9) turns then $e^{-\mu r}$ into $e^{-\mu(\cos (\alpha)+i \sin (\alpha)) r}$. Therefore for $\alpha>0$ the range of the potential will increase, which is counterproductive. This can be avoided by generalizing (9) to complex $\alpha$ 's. Then

$$
e^{-\mu r} \rightarrow \exp \left\{-\mu e^{-\operatorname{Im}(\alpha)}[\cos (\operatorname{Re}(\alpha))+i \sin (\operatorname{Re}(\alpha))] r\right\}
$$

which for $\operatorname{Im}(\alpha)<0$ increases the mass $\mu \cos (\operatorname{Re}(\alpha))$ in the exponent to $\mu e^{-\operatorname{Im}(\alpha)} \cos (\operatorname{Re}(\alpha))$ and thus choosing $\operatorname{Im}(\alpha)$ suitably decreases the range of the interaction again. At the same time also the wave function decreases faster due to the factor $e^{-\operatorname{Im}(\alpha)}$ in the exponent. Let us call that transformation extended CSM (ECSM).

We firstly illustrate the power of the ECSM in the $2 \mathrm{~N}$ system using the Reid $93 \mathrm{NN}$ potential in the state ${ }^{3} P_{0}$. As we have seen in Table \&, an enhancement factor $\lambda_{V}=5.5$ leads to a resonance state with a complex energy eigenvalue $E=(1.42-i 0.75) \mathrm{MeV}$. If one uses in Eq.(8) the first term with $m=0$ only the energy eigenvalue is badly presented (see Tables प and III). The result is improved and we can gain the correct result with the required

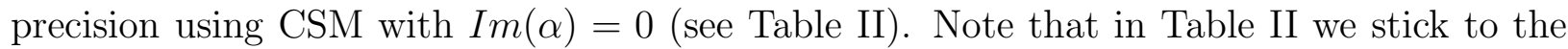
asymptotic form $(m=0)$ in view of the fact that for 3 bodies we also have only approximate 
forms. If we now allow for negative $\operatorname{Im}(\alpha)$ 's then as can be seen in Table II we gain the correct eigenvalue even choosing a very small matching radius.

We also see from Table $\square$ that CSM for increasing $\operatorname{Re}(\alpha)$ gets numerically unstable at a certain value of $\operatorname{Re}(\alpha)$. In this example it happens around $40^{\circ}$. This behavior is intricately connected to the present day parametrizations of NN interactions and puts into question the applicability of CSM for finding broad resonances in nuclear systems. The parametrizations are typically of Yukawa type (one boson exchanges) like in the Reid93 [29] or Reid soft core potential [30]. The analytical continuation to complex r-values leads to an oscillating behavior of the potential, which drastically increase with increasing $\operatorname{Re}(\alpha)$, with unstable numerics as a consequence. We examplify this behavior in Fig. [1 for the Reid93 potential. We checked several of the existing NN potentials and found always this problem ( potentials according to Gogny [31], R.de Tourreil and D.W.L. Sprung [32], H. Eikemeier and H.H. Hackenbroich [33], and R. de Tourreil [34]). In case of the AV14 [35] and AV18 [36] potentials the situation is even worse due to a singularity for complex r-values in their parametrization of the hard core part. Also Gauss-parametrizations are not an exception. In this case the form $\exp \left(-a r^{2}\right)$ limits the complex scaling angle $\operatorname{Re}(\alpha)$ to $\operatorname{Re}(\alpha)<45^{\circ}$.

One possibility to generalize the CSM in order to avoid the above problems is the so called "smooth-exterior" complex scaling (SECS) [26]. The idea is to perform the complex rotation only outside the region of interaction. In this way the analytical continuation of the potential into the complex coordinate plane is avoided. In practice this can be achieved for instance by performing the following transformation (note the printing errors in 26] leading to false expressions (2.1.30) and (2.1.32))

$$
r \rightarrow r^{\prime}=F(r) \rightarrow r e^{i \alpha} \quad \text { for } \quad r \rightarrow \infty
$$

with

$$
F(r)=r+[\exp (i \alpha)-1]\left\{r+\frac{1}{4 \lambda} \ln \frac{\left[1+\exp \left(2 \lambda\left(r-r_{0}\right)\right)\right]\left[1+\exp \left(-2 \lambda\left(r-r_{0}\right)\right)\right]}{\left[1+\exp \left(2 \lambda\left(r+r_{0}\right)\right)\right]\left[1+\exp \left(-2 \lambda\left(r+r_{0}\right)\right)\right]}\right\}
$$

This transformation leads to the smooth-exterior-scaled Hamiltonian 


$$
H=-\frac{\hbar^{2}}{m} \frac{1}{f^{2}(r)} \frac{d^{2}}{d r^{2}}+V(F(r))+\frac{\hbar^{2}}{m} \frac{f^{\prime}(r)}{f^{3}(r)} \frac{d}{d r}+\frac{\hbar^{2}}{m} \frac{l(l+1)}{F^{2}(r)}
$$

with

$$
\begin{aligned}
& f(r)=\frac{d F}{d r}=1+[\exp (i \alpha)-1] g(r) \\
& g(r)=1+0.5\left[\tanh \left(\lambda\left(r-r_{0}\right)\right)-\tanh \left(\lambda\left(r+r_{0}\right)\right)\right]
\end{aligned}
$$

and

$$
\frac{d f}{d r}=0.5 \lambda[\exp (i \alpha)-1]\left[\cosh ^{-2}\left(\lambda\left(r-r_{0}\right)\right)-\cosh ^{-2}\left(\lambda\left(r+r_{0}\right)\right)\right]
$$

The case $\lambda=0$ corresponds to usual CSM $r^{\prime}=r e^{i \alpha}$ [26]. Taking $r_{0}$ outside the region of the NN interaction and choosing a proper value for $\lambda$ a smooth-exterior-scaling path results, which avoids the oscillating changes of the NN potential. We present in Table [II the effectiveness of this approach by aplying it to the ${ }^{1} D_{2}$ resonance state for the Reid93 NN potential with $\lambda_{V}=5.0$. It is enough to keep only the first term in the boundary condition (8) to reproduce the correct resonance position when a sufficiently large value of the scaling angle $\alpha$ is chosen. Now, however, contrary to CSM, no numerical problems arise when large values of $\alpha$ are used. In spite of its success in the $2 \mathrm{~N}$ system we think that the SECS cannot be easily applied in $3 \mathrm{~N}$ systems. We could not overcome problems arising from the permutation operator (see Section III).

\section{THREE-BODY RESONANCES}

Now we investigate 3 -body resonances as solutions of configuration space Faddeev equations. They are often called Kowalski-Noyes equations and have the form [37]

$$
\left(H_{0}+V-E\right) \psi=-V P \psi
$$

Here $H_{0}$ is the kinetic energy, $\mathrm{V}$ the two-body force, $\psi$ a Faddeev component and $\mathrm{P}$ the sum of two permutation operators such that the total, properly symmetrized wave function is $\Psi=(1+P) \psi$. Based on standard Jacobi variables $\vec{x}=\overrightarrow{r_{2}}-\overrightarrow{r_{3}}, \vec{y}=\frac{1}{3}\left(\overrightarrow{x_{1}}-\frac{1}{2}\left(\overrightarrow{x_{2}}+\overrightarrow{x_{3}}\right)\right)$ 
it is convenient to introduce polar coordinates $x \equiv \rho \cos \theta$ and $y \equiv \frac{\sqrt{3}}{2} \rho \sin \theta$. If we express the Faddeev component $\psi(\vec{x}, \vec{y})$ in terms of partial wave amplitudes $\mid \alpha>$ which specify angular momenta and isospin quantum numbers, then we obtain

$$
\psi(\vec{x}, \vec{y})=\sum_{\alpha} \frac{\phi_{\alpha}(x, y)}{x y}<\hat{x} \hat{y} \mid \alpha>
$$

and Eq.(16) turns into a coupled set of partial-integrodifferential equations [28]

$$
\begin{aligned}
\left(\triangle_{\alpha}+\frac{m}{\hbar^{2}} E\right) \phi_{\alpha}(\rho, \theta) & -\sum_{\beta} V_{\alpha \beta}(\rho \cos \theta) \phi_{\beta}(\rho, \theta)=\sum_{\beta} V_{\alpha \beta}(\rho \cos \theta) \\
& \times \sum_{\gamma} \int_{\theta_{-}}^{\theta+} K_{\beta \gamma}\left(\theta, \theta^{\prime}\right) \phi_{\gamma}\left(\rho, \theta^{\prime}\right) d \theta^{\prime}
\end{aligned}
$$

with

$$
\triangle_{\alpha} \equiv \frac{\partial^{2}}{\partial \rho^{2}}+\frac{1}{\rho} \frac{\partial}{\partial \rho}+\frac{1}{\rho^{2}} \frac{\partial^{2}}{\partial \theta^{2}}-\frac{l(l+1)}{\rho^{2} \cos ^{2} \theta}-\frac{\lambda(\lambda+1)}{\rho^{2} \sin ^{2} \theta}
$$

and $\theta^{-}=\left|\theta-\frac{\pi}{3}\right|, \theta^{+}=|| \theta-\frac{\pi}{6}\left|-\frac{\pi}{2}\right|$.

The coupling results from the permutation operator $\mathrm{P}$ and from possible spin dependencies in $\mathrm{V}$. The evaluation of the permutation operator matrix elements $<x y \alpha|P| x^{\prime} y^{\prime} \alpha^{\prime}>$ and the resulting expression for the kernel $K_{\beta \gamma}\left(\theta, \theta^{\prime}\right)$ is presented in Appendix A.

Similarily as in the 2-body case the resonance conditions are such that $\psi(x, y) \equiv \frac{\phi(x, y)}{x y}$ has to be regular at the origin and purely outgoing in all channels. For the reduced amplitudes $\phi(\rho, \theta)$ this amounts to 28,38

$$
\phi(0, \theta)=\phi(\rho, 0)=\phi\left(\rho, \frac{\pi}{2}\right)=0
$$

and for large distances

$$
\phi(\rho, \theta) \rightarrow u(x) e^{i q y} f_{0}+\frac{e^{i k \rho}}{(k \rho)^{\frac{1}{2}}}\left(A(\theta)+\frac{1}{k \rho} B(\theta)+\cdots\right)
$$

The first term in Eq. (21) occurs only in channels $\alpha$, where a two-body bound state $u(x)$ exists. The second part describes the behavior in the 3-body breakup channel. The corresponding momenta are 


$$
\begin{aligned}
q & =\sqrt{m\left(E-E_{2}\right)} \\
k & =\sqrt{m E}
\end{aligned}
$$

where $\mathrm{m}$ is the nucleon mass and $E_{2}$ the two-body binding energy. The boundary conditions (21) apply also to bound states with real eigenvalues E which are located below all two-body thresholds $E_{2}$. For resonances $\mathrm{E}$ is complex and the eigenvalues $\mathrm{E}$ close to the real axis of the physical sheet are of interest. They either lie in second sheets which are accessible to the right of $E_{2}$-values (two-body fragmentation cuts) or in the lower half plane of a second sheet, which is accessible through the $3 \mathrm{~N}$-breakup cut to the right of $E=0$. For a recent description of the cut-structure see for instance [24].

An efficient method for solving Eq. (18) is very well displayed in [28]. One chooses suitable grid points in $\rho$ and $\theta$ and interpolates $\phi(\rho, \theta)$ in terms of cubic splines. Similarily as in the 2-body case the boundary conditions (20) and (21) can be conveniently incorporated either by the absence of certain expansion coefficients in the spline expansion or by suitable relations among the coefficients. Then choosing appropriate collocation points in $\rho$ and $\theta$ one ends up in a homogeneous algebraic set of equations. For realistic NN forces the dimension of the matrix is very large and the tensor product method [39] is a key-technique. We use a Lanczos type iteration method [40] and of course take advantage of the sparceness property of the matrices. It is advantegeous to work with further reduced amplitudes $\mathrm{F}$ defined as $\phi(\rho, \theta) \equiv \rho e^{i k \rho} F(\rho, \theta)$, if one treats resonances below the cut with $R e E>0$. In Appendix $\mathrm{B}$ we present the details of the above procedure.

The main problem in solving Eq. (18) is the approximate nature of the asymptotic form (21). Ab initio it is not known at which $\rho$-value this form is valid with sufficient accuracy. For a recent discussion see [38. Also it is a priori not known how many terms should be included in the asymptotic expansion (21) for a particular $3 \mathrm{~N}$ resonance state. One suspects of course that including more terms one approaches more closely the exact boundary condition. The importance of the terms in Eq.(21) going with increasing powers of $\frac{1}{k \rho}$ depends not only on the resonance energy and the matching value for $\rho$ but also on the magnitudes of the a priori 
unknown functions $A(\theta), B(\theta), \cdots$. For resonances commonly these problems are expected to be overcome using the complex scaling method (CSM) [25]. However, due to the restrictions imposed on this method by the behavior of the NN forces as discussed in Section II we found that this method can be applied in the framework of the configuration space Faddeev equations only for rather narrow resonances. Thereby all relative coordinates $(\mathrm{x}$ and $\mathrm{y}$ in the 3 -body case) are rotated into the complex plane. This amounts to $\rho \rightarrow \rho^{\prime}=\rho e^{i \alpha}$ where $\alpha$ is restricted to rather small values. An example for realistic nn forces is mentioned at the end of this Section. However, if the forces are very simple, CSM can work as will be illustrated below.

One is intended to hope that SECSM which proved to be very efficient in the 2-body case will help here, too. However, according to our insights up to now this approach leads to problems we could not solve when calculating the permutation operator $\mathrm{P}$ and when performing the transition from cartesian to polar relative coordinates.

The only possibility left is to look at solutions of Eq.(18) without leaving the real r-axis and taking the boundary condition (21) with a sufficient number of terms into account in order to reach as close as possible the exact boundary condition. In this manner one can hope to get convergence for the resulting resonance position.

We checked our configuration space code by calculating the energy of the bound state of ${ }^{3} \mathrm{H}$ nuclei with the Reid $93 \mathrm{NN}$ potential taking different NN force components into account. In Table IV we present our numbers together with results of momentum space Faddeev calculations [41]. The very good agreement between both approaches is clearly seen.

In the first step we considered 3 bosons interacting via Gaussian S-wave forces

$$
V(r)=-120 \exp \left(-r^{2}\right)+12 \exp (-r / 3)^{2} \quad[M e V] .
$$

They are such that a barrier is built in, which is an obvious and trivial mechanism generating a resonance. That interaction does not support a two-body bound state and in the asymptotic form (21) the first term is absent. For 3 bosons in the $J^{\pi}=0^{+}$state 
and interacting by S-wave forces only the kernel $K_{\alpha \beta}=\frac{1}{4 \pi}$. This model has been used in 42 in applying the stochastic variational method. The resonance energy found there is $E=(8.60-i 1.84) \mathrm{MeV}$. In this case CSM works. For instance for a matching radius of $\rho=10 \mathrm{fm}$ and taking the A- and B-terms in (21) into account we achieved the results shown in Table $\nabla$. For $\operatorname{Re}(\alpha)=40.0^{\circ}$ we are close to the result found in [42] but not close enough. A smaller matching radius, however, like $\rho=5 \mathrm{fm}$ does not work. The correct result can however be achieved if in addition we turn on $\operatorname{Im}(\alpha)<0$. This is demonstrated in Table $\square$. We illustrate in Figs.2 and 3 typical resonance amplitudes $\mathrm{F}$ working with real coordinates or with complex coordinates. In both cases the matching radius has been chosen as $5 \mathrm{fm}$. In the first case this is not sufficient to achieve the correct complex energy eigenvalue, whereas in the second case the amplitude is already sufficiently damped at $\rho=5 \mathrm{fm}$ and the correct energy eigenvalue is accessible at such a small matching radius.

In this simple case one can study the problem how many terms $A, B, \cdots$ in the boundary condition (21) should be taken into account to get the correct resonance position. Of course it will depend on the value of the matching radius $\rho$. We present in Table $\nabla$ resonance positions obtained with increasing number of terms in the boundary condition (21) and different matching radii. For the details to include arbitrary number of terms in the asymptotic condition we refer to Appendix B. It is seen from Table V1 that for matching radii $>10 \mathrm{fm}$ it is practically sufficient to take only the two leading terms going with A and B into account in order to get the correct resonance position. We also see that the restriction to the A term alone is totally insufficient. Unfortunately this example is far from reality in nuclear physics. It does not contain a short range repulsion and the mechanism for creating a resonance in few nucleon systems is different in reality, where it is not caused by a barrier in the potential itself.

Now we investigate the case of three interacting neutrons in states with different total angular momenta and parity. We allowed the nn force to act in the states ${ }^{1} S_{0},{ }^{3} P_{0}$ and ${ }^{3} P_{2}-{ }^{3} F_{2}$. They are of attractive nature while the repulsive ones were neglected. This is only a feasibility study and should provide a first orientation. In the first step the nn 
forces were chosen as a smooth, local NN force of ref. [31], called in the following Gogny potential. It is a rather simple approach to NN phase-shifts through forces of central, tensor and spin-orbit type supplemented by a $L_{12}$ term, all of which of Gaussian form. To initiate the search process for the position of the resonance energy we artificially enhanced the force components in the three states such that all of them support a two-neutron bound state at the same energy. An example is shown in Table VII for a $J^{\pi}=3 / 2^{-}$state of three neutrons. For these enhancement factors also the $3 \mathrm{n}$ system is bound. Then we reduce the enhancement factors (some intermediate cases are shown in Table VII), which traces out a bound state trajectory. In this case we see that the $3 \mathrm{n}$ bound state still exists even when all $2 \mathrm{n}$ subsystem bound states have disappeared. Then we can neglect the first term in the boundary condition (21). Decreasing further the enhancement factor(s) (in this specific case only for ${ }^{3} P_{2}-{ }^{3} F_{2}$ ) the energy eigenvalue goes into the second sheet and traces out a resonance trajectory. The search for the resonance position corresponding to the actual nn force is finished when the enhancement factors in all nn force components have reached the values $\lambda_{i}=1.0$. As we shall see in the case of three neutrons we face numerical instabilities before all $\lambda^{\prime} s$ reach the value 1 . We can present the resonance trajectory only partially up to the point, where we encounter those instabilities. We expect that they are probably caused by the difficulty to fulfill the resonance condition, which requires that the exponentially decreasing, purely incoming wave is much smaller than the exponentially increasing purely outgoing wave. As we see from Table VII the $J^{\pi}=3 / 2^{-} 3 \mathrm{n}$ bound state disappears when the enhancement factors arrive at $\left(\lambda_{{ } S_{0}}, \lambda_{3} P_{0}, \lambda^{3} P_{2}-{ }^{3} F_{2}\right)=(1.0,1.0,4.35)$. In Table VIII the trajectory of the $3 / 2^{-}$3n resonance in the second energy sheet is mapped out by decreasing the enhancement factor $\lambda^{3} P_{2}-{ }^{3} F_{2}$ in step of 0.05 starting from $\lambda_{3 P_{2}-{ }^{3} F_{2}}=4.3$. The final reliable position reached is for $\lambda^{3} P_{2}-^{3} F_{2}=3.6$ with the value $E_{3 n}^{3 / 2^{-}}=(5.30-i 4.67) \mathrm{MeV}$. As can be seen this stable position is only obtained when at least 4 terms in the boundary condition (21) are taken into account. We checked that our particular distribution of $\rho$ and $\theta$ points as well as the choice of the matching radius in $\rho$ does not influence the resulting trajectory. The values given in Table VIII are based on a matching radius $\rho=30.0 \mathrm{fm}$ and 
typically $N_{\rho}=30$ and $N_{\theta}=25$ points distributed by scaling factors $S_{\rho}=1.2$ and $S_{\theta}=0.9$ (see ref. [28]). It is unfortunate that right now we are not able to reach the final position with $\lambda_{3} P_{2}-{ }^{3} F_{2}=1$. However very likely the width will be quite large. Therefore one cannot expect to see a visible enhancement around some $3 \mathrm{n}$ c.m. energy in a reaction producing three neutrons in the state $3 / 2^{-}$.

Similarily to $3 / 2^{-}$behave the $1 / 2^{-}$and $3 / 2^{+}$states based on the Gogny potential. With proper enhancement factors $\lambda_{V}$ the three neutrons are bound together with 2-neutron bound states for ${ }^{1} S_{0},{ }^{3} P_{0}$ and ${ }^{3} P_{2}-{ }^{3} F_{2}$. These $3 n$ states remain bound even when the 2-neutron bound states disappear with appropriately reduced enhancement factors. They disappear into the second energy sheet when the enhancement factors reach $(1.0,7.5,4.75)$ and $(1.0,5.5,4.8)$ for $J^{\pi}=1 / 2^{-}$and $3 / 2^{+}$, respectively. These enhancements factors are of course not unique but we worked with those examples. Following their trajectories before numerical instabilities set in results in the complex energy eigenvalues shown for the Gogny potential in Table [X]. For the $1 / 2^{-}$and $3 / 2^{+}$states, similarily as for $3 / 2^{-}$, the final, converged position is obtained only when at least 4 terms in the boundary condition are taken into account. In Fig. 1 we show the trajectories for the three states $1 / 2^{-}, 3 / 2^{-}$, and $3 / 2^{+}$ for the Gogny potential. We note that the trajectories are not unique and depend how the enhancement factors are changed. Our startegy was to bring all of them together to one as close as possible.

In this article we did not investigate the state $1 / 2^{+}$, since we found, like in [9] that the $3 \mathrm{n}$ bound state disappears earlier than the $2 \mathrm{n}$ bound states. In other words, the $3 \mathrm{n}$ bound state trajectory will enter into the $2 \mathrm{n}-\mathrm{n}$ two-body fragmentation cut. We left that study with the additional asymptotic term in Eq.(21) to a future investigation.

It is seen from Table $\mathbb{I X}$ that the $3 / 2^{+}$state for the Gogny potential is relatively narrow. Taking the enhancement factors $\lambda_{i}=(1.0,5.3,4.6)$ results in a resonance position $E_{3 n}^{3 / 2^{+}}=$ $(2.88-i 0.23) \mathrm{MeV}$. For such a narrow resonance the CSM works properly for not too large values of the $\alpha$ angle (see Table $\mathrm{X}$ ). Increasing it, however, the resulting energy eigenvalue is totally wrong what can be traced back to the increasing oscillations of the NN potential 
resulting from its analytical continuation to the complex r-values.

In the last step we checked how the results depend on the NN interaction used. We choose a modern, realistic NN interaction as given by the Reid 93 potential. The resulting positions of the $3 \mathrm{n}$ resonances, as far as we could trace them out, are shown for two examples

in Table XI. In spite of the fact that the particular values of the $\lambda_{V}$ 's change of course, the resulting picture is basically the same as for the Gogny potential. We are still too far away from all $\lambda_{i}=1$ in order to definitely say that all the widths will be quite large. But this will likely be the case. Again to achieve converged results one has to take at least 4 terms in the boundary condition (21) into account.

\section{SUMMARY}

We investigated the configuration space Faddeev equations with the aim to determine the location of 3-neutron resonances. Realistic nn forces have been used. The asymptotic form in the $3 \mathrm{n}$ breakup channel is known only in the sense of an asymptotic expansion. We found that several terms thereof have to be included in order to get stable and reliable results, which are independent of the matching radius. The search for the resonance positions was carried through by artificially enhancing $2 \mathrm{n}$ force components such that a $3 \mathrm{n}$ bound state exists. Then by reducing those enhancement factors we followed the paths of the energy eigenvalue into the second sheet adjacent to $\operatorname{Re}(E) \geq 0$. Unfortunately it turned out that numerical instabilities set in before we reached the actual strengths factors 1 for all the individually enhanced force components. Therefore we could not reach the very final positions of the studied $3 \mathrm{n}$ resonances in the states $J^{\pi}=1 / 2^{-}, 3 / 2^{-}$, and $3 / 2^{+}$. The detailed reason for those instabilities remains to be found out. Despite of that our results already indicate that the imaginary parts of the complex resonance energies will be quite large, so that one should not expect to see resonances in experiments.

We also applied the CSM, which works beautifully in atomic phyics. The complex coordinates turn the exponentially increasing resonance wave function into an exponentially 
decreasing one, like for a bound state, and the problem with the approximate boundary conditions is avoided. However, for realistic NN interactions the complex scaling transformation $r \rightarrow r e^{i \alpha}$ increases the interaction range by a factor $\frac{1}{\cos \alpha}$ and leads for scaling angles $\alpha$ greater than about $40^{\circ}$ to a strong oscillations of the potential. This restricts the application of the complex scaling method in more than 2-nucleon systems to rather narrow resonances. In the 2-nucleon system one can avoid the above restriction by performing the complex rotation outside the region of non-zero interaction. Due to the neccessary particle permutation in the 3-body system we were not able to use that idea in the 3-body context.

This project deserves further investigations to locate all lowest resonances in the three nucleon systems based on modern NN and possibly three-nucleon forces.

\section{Acknowledgements}

This work was supported by the Deutsche Forschungsgemeinschaft under Project No. G187/24-1. The numerical calculations have been performed on the CRAY T90 and the CRAY T3E of the Höchstleistungsrechenzentrum in Jülich, Germany. 


\section{REFERENCES}

[1] D.R. Tilley, H.R. Weller, ann H.H. Hasan, Nucl.Phys. A474, 1 (1987).

[2] M.Yuly et al, Phys. Rev. C55, 1884 (1997).

[3] J. Sperinde, D. Frederickson, R.Hinkins, V.Prez-Mendez, and B. Smith, Phys.Lett. B32, $185(1970)$.

[4] L.E. Williams, C.J. Batty, B.E. Bonner, C. Tschalär, H.C. Benöhr, and A.S. Clough, Phys.Rev.Lett. 23, 1181 (1969).

[5] A. Setz et al, Nucl. Phys. A457, 669 (1986).

[6] D.V. Aleksandrov, E.Yu. Nikol'skii, B.G. Novatskii, and D.N. Stepanov, JETP Lett. 59, 320 (1994).

[7] A. Csótó, H. Oberhummer, and R. Pichler, Phys. Rev. C53, 1589 (1996).

[8] R. Offermann and W. Glöckle, Nucl. Phys. A318, 138 (1979).

[9] W. Glöckle, Phys. Rev. C18, 564 (1978).

[10] A. Delfino, W. Glöckle, Phys. Rev. C30, 376 (1984).

[11] S.A. Sofianos, S.A. Rakityansky, and G.P. Vermark, J. Phys. G: Nucl. Part. Phys. 23, 1619 (1997).

[12] A.K. Motovilov, Math. Nachr. 187, 147 (1997).

[13] A.K. Motovilov, E.A. Kolganova, in Proc. of 16th European Conference on Few-Body Problems in Physics, 1-6 June 1998, Autrans, France, to appear in Few-Body Systems Supplement.

[14] W. Glöckle, H. Witała, D. Hüber, H. Kamada, J. Golak, Phys. Rep. 274, 107 (1996).

[15] A. Nogga, D. Hüber, H. Kamada, and W. Glöckle, Phys. Lett. B409, 19 (1997). 
[16] C.R. Chen, G.L. Payne, J.L. Friar, and B.F. Gibson, Phys. Rev. C33, 1740 (1986).

[17] J. Carlson, R. Schiavilla, Rev. Mod. Phys. 70, 743 (1998).

[18] M. Viviani, Nucl. Phys. A 631, 111c (1998).

[19] Y. Wu, S. Ishikawa, T. Sasakawa, Few-Body Systems 15, 145 (1993).

[20] J.L. Friar, G.L. Payne, W. Glöckle, D. Hüber, H. Witała, Phys. Rev. C51, 2356 (1995).

[21] A. Kievsky, M. Viviani, S. Rosati, D. Hüber, W. Glöckle, H. Kamada, H. Witała, and J. Golak, Phys. Rev. C58, 3085 (1998).

[22] S.P. Merkuriev, C. Gignoux, A. Laverne, Ann. Phys. 99, 30 (1976).

[23] W. Glöckle, Z. Phys. 271, 31 (1974).

[24] E.A. Kolganowa and A.K. Motovilow, Phys. Atom. Nucl. 60, 177 (1977).

[25] Y.K. Ho, Phys. Rep. 99, 1 (1983).

[26] N. Moiseyev, Phys. Reports, 302, 211 (1998).

[27] W. Glöckle, The Quantum Mechanical Few-Body Problem, Berlin - Heidelberg - New York, Springer 1983.

[28] G.L. Payne, Lecture Notes in Physics, 273, 64 (1987).

[29] V. G. J. Stoks, R.A.M. Klomp, C.P.F. Terheggen, and J.J. de Swart, Phys. Rev. C 49, 2950 (1994).

[30] R.V.Reid, Ann.Phys.(N.Y.), 50, 411 (1968).

[31] D. Gogny, P. Pires and R. de Tourreil, Phys. Lett. 32B, 591 (1970).

[32] R.de Tourreil and D.W.L. Sprung, Nucl. Phys. A201, 193 (1973).

[33] H. Eikemeier and H.H. Hackenbroich, Nucl.Phys. A169, 407 (1971). 
[34] R. de Tourreil et al., Nucl.Phys., A242, 445 (1975).

[35] R.B.Wiringa, R.A. Smith, T.L. Ainsworth, Phys. Rev. C29, 1207 (1984).

[36] R.B. Wiringa, V.G.J. Stoks, and R. Schiavilla, Phys. Rev. C51, 38 (1995).

[37] H.P. Noyes, in Three Body Problems in Nuclear and Particle Physics, edited by J.S.C. McKee and P.M. Rolph (North-Holland, Amsterdam, 1970).

[38] W. Glöckle, G.L. Payne, Phys. Rev. C45, 974 (1992).

[39] N.W. Schellingerhout, PhD thesis, Groningen University, 1995.

[40] W. Saake, Diploma thesis, Bochum University, 1992, unpublished.

[41] A. Nogga, private communication.

[42] N. Tanaka, Y. Suzuki, and K. Varga, Phys. Rev. C56, 562 (1997). 


\section{APPENDIX A: PERMUTATION OPERATOR}

To evaluate the term on the right hand side of Eq.(16) one needs

$$
<x y \beta|P| \psi>=\sum_{\gamma} \int d x^{\prime} x^{\prime 2} d y^{\prime} y^{\prime 2}<x y \beta|P| x^{\prime} y^{\prime} \gamma>\frac{\phi_{\gamma}\left(x^{\prime}, y^{\prime}\right)}{x^{\prime} y^{\prime}}
$$

where the permutation operator is $P \equiv P_{12} P_{23}+P_{13} P_{23}$ and the set of discrete quantum numbers abbreviated by $\beta$ or $\gamma$ is $\left\{(l s) j(\lambda 1 / 2) I(j I) J M ;(t 1 / 2) T M_{T}\right\}$. Here $1, \mathrm{~s}$, and $\mathrm{j}$ are the orbital angular momentum, total spin and total angular momentum in the two-body subsystem $2-3, \lambda, 1 / 2$, and I are the corresponding quantities for particle 1 , and JM denote the conserved $3 \mathrm{~N}$ total angular momentum and its magnetic quantum number. In isospin space, $t$ refers to particles 2 and 3 and couples with the isospin $1 / 2$ of particle 1 to the total isospin $\mathrm{T}$ and its magnetic quantum number $M_{T}$. Following [27] one obtains for the matrix element of the permutation operator

$$
<x y \alpha|P| x^{\prime} y^{\prime} \alpha^{\prime}>=\int_{-1}^{1} d u \frac{\delta\left(x^{\prime}-\tilde{x}^{\prime}\right)}{\left(x^{\prime}\right)^{l^{\prime}+2}} \frac{\delta\left(y^{\prime}-\tilde{y}^{\prime}\right)}{\left(y^{\prime}\right)^{\lambda^{\prime}+2}} \tilde{G}_{\alpha \alpha^{\prime}}^{c a r t}(x, y, u)
$$

with

$$
\begin{aligned}
& \tilde{x}^{\prime}=\sqrt{\frac{1}{4} x^{2}+x y u+y^{2}} \\
& \tilde{y}^{\prime}=\sqrt{\frac{9}{16} x^{2}-\frac{3}{4} x y u+\frac{1}{4} y^{2}}
\end{aligned}
$$

and

$$
\tilde{G}_{\alpha \alpha^{\prime}}^{c a r t}(x, y, u)=\sum_{k} P_{k}(u) \sum_{r_{1}+r_{2}=l^{\prime}} \sum_{s_{1}+s_{2}=\lambda^{\prime}}(x)^{r_{1}+s_{1}}(y)^{r_{2}+s_{2}} \tilde{g}_{\alpha \alpha^{\prime}}^{r_{1} s_{1} k}
$$

The purely geometrical quantity $\tilde{g}_{\alpha \alpha^{\prime}}^{r_{1} s_{1} k}$ is given by

$$
\begin{aligned}
\tilde{g}_{\alpha \alpha^{\prime}}^{r_{1} s_{1} k}= & -\sqrt{\hat{l} \hat{l}^{\prime} \hat{s} \hat{s}^{\prime} \hat{j} \hat{j}^{\prime} \hat{\lambda} \hat{\lambda} \hat{\lambda}^{\prime} \hat{I} \hat{I}^{\prime} \hat{t} \hat{t}^{\prime}}(-1)^{k+l^{\prime}+s_{1}} \hat{k}\left(\frac{1}{2}\right)^{r_{1}+s_{2}}\left(\frac{3}{4}\right)^{s_{1}} \\
& \sum_{L S}(-1)^{L} \hat{L} \hat{S}\left\{\begin{array}{ccc}
l & s & j \\
\lambda & \frac{1}{2} & I \\
L & S & J
\end{array}\right\}\left\{\begin{array}{ccc}
l^{\prime} & s^{\prime} & j^{\prime} \\
\lambda^{\prime} & \frac{1}{2} & I^{\prime} \\
L & S & J
\end{array}\right\}\left\{\begin{array}{ccc}
1 / 2 & 1 / 2 & t \\
1 / 2 & T & t^{\prime}
\end{array}\right\}\left\{\begin{array}{ccc}
1 / 2 & 1 / 2 & s \\
1 / 2 & S & s^{\prime}
\end{array}\right\}
\end{aligned}
$$




$$
\begin{aligned}
& \sqrt{\frac{\hat{l^{\prime} !}}{\left(2 r_{1}\right) !\left(2 r_{2}\right) !}} \sum_{r_{3}} \hat{r}_{3}\left(\begin{array}{ccc}
r_{1} & s_{1} & r_{3} \\
0 & 0 & 0
\end{array}\right)\left(\begin{array}{ccc}
l & r_{3} & k \\
0 & 0 & 0
\end{array}\right) \\
& \sqrt{\frac{\hat{\lambda}^{\prime} !}{\left(2 s_{1}\right) !\left(2 s_{2}\right) !}} \sum_{s_{3}} \hat{s}_{3}\left(\begin{array}{ccc}
r_{2} & s_{2} & s_{3} \\
0 & 0 & 0
\end{array}\right)\left(\begin{array}{ccc}
\lambda & s_{3} & k \\
0 & 0 & 0
\end{array}\right) \\
& \left\{\begin{array}{ccc}
l & r_{3} & k \\
s_{3} & \lambda & L
\end{array}\right\}\left\{\begin{array}{lll}
r_{1} & r_{2} & l^{\prime} \\
s_{1} & s_{2} & \lambda^{\prime} \\
r_{3} & s_{3} & L
\end{array}\right\}
\end{aligned}
$$

The superscript cart stands for cartesian and $\hat{l} \equiv 2 l+1$. To arrive at the kernel $K_{\beta \gamma}$ on the right-hand side of Eq.(18) one has to evaluate

$$
x y<x y \beta|P| \psi>=\sum_{\gamma} x y \int_{-1}^{1} d u \frac{\tilde{G}_{\beta \gamma}^{c a r t}(x, y, u)}{\tilde{x}^{l_{\gamma}} \tilde{y}^{\prime \lambda_{\gamma}}} \frac{\phi_{\gamma}\left(\tilde{x}^{\prime}, \tilde{y}^{\prime}\right)}{\tilde{x}^{\prime}, \tilde{y}^{\prime}}
$$

We introduce polar coordinates

$$
\begin{aligned}
& \tilde{x}^{\prime} \equiv \rho \cos \theta^{\prime} \\
& \tilde{y}^{\prime} \equiv \frac{\sqrt{3}}{2} \rho \sin \theta^{\prime}
\end{aligned}
$$

and changing the $u$ to the $\theta^{\prime}$ integration

$$
\int_{-1}^{1} d u \frac{x y}{\tilde{x}^{\prime} \tilde{y}^{\prime}} \cdots=\frac{4}{\sqrt{3}} \int_{\theta^{-}}^{\theta^{+}} d \theta^{\prime} \cdots
$$

one arrives at

$$
x y<x y \beta|P| \psi>=\sum_{\gamma} \frac{4}{\sqrt{3}} \int_{\theta^{-}}^{\theta^{+}} d \theta^{\prime} \frac{\tilde{G}_{\beta \gamma}^{\text {polar }}\left(\theta, \theta^{\prime}\right)}{\left(\cos \theta^{\prime}\right)^{l_{\gamma}}\left(\frac{\sqrt{3}}{2} \sin \theta^{\prime}\right)^{\lambda_{\gamma}}} \phi_{\gamma}\left(\rho, \theta^{\prime}\right)
$$

with

$$
\tilde{G}_{\beta \gamma}^{\text {polar }}\left(\theta, \theta^{\prime}\right)=\sum_{k} P_{k}\left(u\left(\theta, \theta^{\prime}\right)\right) \sum_{r_{1}+r_{2}=l_{\gamma}} \sum_{s_{1}+s_{2}=\lambda_{\gamma}}(\cos \theta)^{r_{1}+s_{1}}\left(\frac{\sqrt{3}}{2} \sin \theta\right)^{r_{2}+s_{2}} \tilde{g}_{\beta \gamma}^{r_{1} s_{1} k}
$$

and

$$
u\left(\theta, \theta^{\prime}\right)=\frac{\cos ^{2} \theta^{\prime}-\frac{1}{4} \cos ^{2} \theta-\frac{3}{4} \sin ^{2} \theta}{\frac{\sqrt{3}}{2} \cos \theta \sin \theta}
$$

From Eq.(A9) follows that

$$
K_{\beta \gamma}\left(\theta, \theta^{\prime}\right)=\frac{4}{\sqrt{3}} \frac{\tilde{G}_{\beta \gamma}^{\text {polar }}\left(\theta, \theta^{\prime}\right)}{\left.\left(\cos \theta^{\prime}\right)^{l_{\gamma}\left(\frac{\sqrt{3}}{2}\right.} \sin \theta^{\prime}\right)^{\lambda_{\gamma}}}
$$




\section{APPENDIX B: THE SOLUTION OF THE ENERGY EIGENVALUE PROBLEM INCLUDING N-TERMS IN THE ASYMPTOTIC BOUNDARY CONDITION}

Due to numerical reasons it is advantegous to extract the exponential part from the reduced Faddeev amplitude $\phi_{\alpha}(\rho, \theta)$ and to work instead with

$$
F_{\alpha}(\rho, \theta) \equiv \phi_{\alpha}(\rho, \theta) /\left(\rho e^{i k \rho}\right)
$$

Following ref. [28] one introduces suitable grid points in $\rho\left\{\rho_{i}, \quad i=0, \cdots, I ; \quad \rho_{0}=0, \rho_{I}=\right.$

$\left.\rho_{\max }\right\}$ and in $\theta \quad\left\{\theta_{k}, \quad k=0, \cdots, K ; \quad \theta_{0}=0, \theta_{K}=\frac{\pi}{2}\right\}$. Then one can write $F_{\alpha}(\rho, \theta)$ in terms of cubic splines $s_{m}(\rho)$ and $s_{n}(\theta)$ as

$$
F_{\alpha}(\rho, \theta)=\sum_{m=0}^{M+1} \sum_{n=0}^{N+1} a_{\alpha m n} s_{m}(\rho) s_{n}(\theta)
$$

with $M=2 I$ and $N=2 K$.

Due to the properties of the cubic splines as given in 28] one has

$$
\begin{aligned}
F_{\alpha}\left(\rho_{i}, \theta_{k}\right) & =a_{\alpha, 2 i, 2 k} \\
\left.\frac{\partial F_{\alpha}}{\partial \rho}\right|_{\rho_{i}, \theta_{k}} & =a_{\alpha, 2 i+1,2 k} \\
\left.\frac{\partial F_{\alpha}}{\partial \theta}\right|_{\rho_{i}, \theta_{k}} & =a_{\alpha, 2 i, 2 k+1}
\end{aligned}
$$

The boundary conditions at the origin (20) imply that

$$
\begin{aligned}
a_{\alpha, 0, n} & =0 \\
a_{\alpha, m, 0} & =0 \\
a_{\alpha, m, n=N} & =0 .
\end{aligned}
$$

Writing the asymptotic expansion (21) for $F_{\alpha}(\rho, \theta)$ under the assumption that no two-body bound state exists in any channel $\alpha$ results in

$$
F_{\alpha}(\rho, \theta) \rightarrow \frac{1}{\rho(k \rho)^{1 / 2}} \sum_{l=1}^{N_{t}} \frac{B_{l}^{\alpha}(\theta)}{(k \rho)^{l-1}}
$$

when $N_{t}$ terms are included in the asymptotic form. 
Imposing the boundary condition (B5) at $\rho=\rho_{\max }$ implies the dependence of $a_{\alpha, m=M+1, n}$ on $a_{\alpha, m=M, n}$ and $B_{l}^{\alpha}(\theta)$. In order to remove the unknown $B_{l}^{\alpha}(\theta)$ functions the boundary condition has to be imposed on the last $N_{t}$ points of the $\rho$-grid. This leads to the dependence of $a_{\alpha, m=M+1, n}$ on $a_{\alpha, m=M, n}, \cdots, a_{\alpha, m=M-2\left(N_{t}-1\right), n}$. In the following the form of this dependence is derived.

The asymtotic form for the partial derivative $\frac{\partial F_{\alpha}}{\partial \rho}$ is according to (B5)

$$
\frac{\partial F_{\alpha}(\rho, \theta)}{\partial \rho} \rightarrow-\tilde{A}_{1}(\rho) F_{\alpha}(\rho, \theta)-\sum_{l=2}^{N_{t}} \tilde{A}_{l}(\rho) B_{l}^{\alpha}(\theta)
$$

with

$$
\tilde{A}_{1}(\rho)=\frac{3}{2 \rho}
$$

and for $l \geq 2$

$$
\tilde{A}_{l}(\rho)=\frac{l-1}{\rho^{2}\left(k_{0} \rho\right)^{l-1 / 2}}
$$

Taking the partial derivative of (Bי with respect to $\rho$ and the form (B6) yields for the last $N_{t}$ points of the $\rho$ grid $\left\{\rho=\rho_{I-i+1}, \quad i=1, \cdots, N_{t}\right\}$

$$
\sum_{n} a_{\alpha, M+1-2(i-1), n} s_{n}(\theta)=-\sum_{n} a_{\alpha, M-2(i-1), n} s_{n}(\theta) \tilde{A}_{1}\left(\rho_{I-i+1}\right)-\sum_{l=2}^{N_{t}} \tilde{A}_{l}\left(\rho_{I-i+1}\right) B_{l}^{\alpha}(\theta) .
$$

Eq.(B9) together with its $\theta$-derivative taken at the points of the $\theta$-grid lead to

$$
a_{\alpha, M+1-2(i-1), n}=-a_{\alpha, M-2(i-1), n} \tilde{A}_{1}\left(\rho_{I-i+1}\right)-\sum_{l=2}^{N_{t}} \tilde{A}_{l}\left(\rho_{I-i+1}\right) \tilde{B}_{l}^{\alpha}\left(\theta_{k}\right)
$$

where for $n=2 k \quad \tilde{B}_{l}^{\alpha}=B_{l}^{\alpha}\left(\theta_{k}\right)$ and for $n=2 k+1 \quad \tilde{B}_{l}^{\alpha}=\left.\frac{d B_{l}^{\alpha}}{d \theta}\right|_{\theta=\theta_{k}}$.

The set $(\overline{B 10})$ are $N_{t}$ equations for $N_{t}-1$ unknowns $\tilde{B}_{l}^{\alpha}\left(\theta_{k}\right)$. They allow to write $a_{\alpha, M+1, n}$ in terms of the $a_{\alpha, M-i+1, n}, i=1, \cdots, 2\left(N_{t}-1\right)+1$ as

$$
a_{\alpha, M+1, n}=\sum_{i=1}^{2\left(N_{t}-1\right)+1} a_{\alpha, M-i+1, n} C_{i}
$$

where the $C_{i}$ 's are functions of all the $\tilde{A}_{l}\left(\rho_{I-i+1}\right)$ 's resulting from (B10).

In this way, incorporating the boundary conditions $(\mathbb{B} 4)$ and $(\mathbb{B} 5)$ one can write $F_{\alpha}(\rho, \theta)$ on the chosen grids as 


$$
F_{\alpha}(\rho, \theta)=\sum_{m=1}^{M} \sum_{n=1}^{N} a_{\alpha m n} s_{m}(\rho) s_{n}(\theta)+\sum_{n=1}^{N}\left[\sum_{i=1}^{2\left(N_{t}-1\right)+1} a_{\alpha, M-i+1, n} C_{i}\right] s_{M+1}(\rho) s_{n}(\theta)
$$

where, due to the last equation in $(\mathbb{B} 4)$ we renumbered $n=N+1$ by $n=N$.

Inserting the expansion (B12) with the help of (B1) into Eq. 18$)$ and choosing collocation points $\rho_{p}, \quad p=1, \cdots, 2 I$ and $\theta_{q}, \quad q=1, \cdots, 2 K$ [28] yields

$$
\sum_{\beta=1}^{N_{c}} \sum_{m=1}^{M} \sum_{n=1}^{N} A_{\alpha p q, \beta m n} a_{\beta m n}=\sum_{\beta=1}^{N_{c}} \sum_{m=1}^{M} \sum_{n=1}^{N} B_{\alpha p q, \beta m n} a_{\beta m n}
$$

with

$$
\begin{aligned}
A_{\alpha p q, \beta m n}= & \delta_{\alpha \beta}\left[s_{m}^{\prime \prime}\left(\rho_{p}\right) s_{n}\left(\theta_{q}\right)+\left(\frac{3}{\rho_{p}}+2 i k\right) s_{m}^{\prime}\left(\rho_{p}\right) s_{n}\left(\theta_{q}\right)+\frac{1}{\rho_{p}^{2}} s_{m}\left(\rho_{p}\right) s_{n}^{\prime \prime}\left(\theta_{q}\right)\right. \\
& \left.-\left\{\frac{l(l+1)}{\rho_{p}^{2} \cos ^{2} \theta_{q}}+\frac{\lambda(\lambda+1)}{\rho_{p}^{2} \sin ^{2} \theta_{q}}-\left(\frac{1}{\rho_{p}^{2}}+\frac{3 i k}{\rho_{p}}\right)\right\} s_{m}\left(\rho_{p}\right) s_{n}\left(\theta_{q}\right)\right]
\end{aligned}
$$

and

$$
\begin{aligned}
B_{\alpha p q, \beta m n}= & V_{\alpha \beta}\left(\rho_{p} \cos \theta_{q}\right) s_{m}\left(\rho_{p}\right) s_{n}\left(\theta_{q}\right) \\
& +\sum_{\gamma=1}^{N_{c}} V_{\alpha \gamma}\left(\rho_{p} \cos \theta_{q}\right) s_{m}\left(\rho_{p}\right) \int_{\theta^{-}}^{\theta^{+}} K_{\gamma \beta}\left(\theta_{q}, \theta^{\prime}\right) s_{n}\left(\theta^{\prime}\right) d \theta^{\prime} \\
& +\delta_{m, M-i+1}\left(1 \leq i \leq 2\left(N_{t}-1\right)+1\right)\left\{-\delta_{\alpha \beta} C_{i}\left[s_{M+1}^{\prime \prime}\left(\rho_{p}\right) s_{n}\left(\theta_{q}\right)\right.\right. \\
& +\left(\frac{3}{\rho_{p}}+2 i k\right) s_{M+1}^{\prime}\left(\rho_{p}\right) s_{n}\left(\theta_{q}\right)+\frac{1}{\rho_{p}^{2}} s_{M+1}\left(\rho_{p}\right) s_{n}^{\prime \prime}\left(\theta_{q}\right) \\
& \left.-\left\{\frac{l(l+1)}{\rho_{p}^{2} \cos ^{2} \theta_{q}}+\frac{\lambda(\lambda+1)}{\rho_{p}^{2} \sin ^{2} \theta_{q}}-\left(\frac{1}{\rho_{p}^{2}}+\frac{3 i k}{\rho_{p}}\right)\right\} s_{M+1}\left(\rho_{p}\right) s_{n}\left(\theta_{q}\right)\right] \\
& V_{\alpha \beta}\left(\rho_{p} \cos \theta_{q}\right) s_{M+1}\left(\rho_{p}\right) s_{n}\left(\theta_{q}\right) \\
& \left.+\sum_{\gamma=1}^{N_{c}} V_{\alpha \gamma}\left(\rho_{p} \cos \theta_{q}\right) s_{M+1}\left(\rho_{p}\right) \int_{\theta^{-}}^{\theta^{+}} K_{\gamma \beta}\left(\theta_{q}, \theta^{\prime}\right) s_{n}\left(\theta^{\prime}\right) d \theta^{\prime}\right\}
\end{aligned}
$$

The number of channels is denoted by $N_{c}$. This eigenvalue problem (B13) can be treated in a standard way by changing it into the form

$$
A^{-1} B a=\lambda a
$$

and searching for that energy at which $\lambda=1$.

To solve Eq.(B16) we used a Lanczos type iteration method [40]. For the application of $\mathrm{B}$ and $A^{-1}$ on a given vector a we applied the tensor product method [39]. Hereby $\mathrm{A}$ is decomposed as 


$$
A=\bar{A} \otimes \bar{B}+\bar{C} \otimes \bar{D}
$$

with

$$
\begin{aligned}
\bar{A}_{p m} & \equiv s_{m}\left(\rho_{p}\right)\left(\frac{1}{\rho_{p}^{2}}+\frac{3 i k}{\rho_{p}}\right)+\left(\frac{3}{\rho_{p}}+2 i k\right) s_{m}^{\prime}\left(\rho_{p}\right)+s_{m}^{\prime \prime}\left(\rho_{p}\right) \\
\bar{B}_{q n}^{\alpha \beta} & \equiv \delta_{\alpha \beta} s_{n}\left(\theta_{q}\right) \\
\bar{C}_{p m} & \equiv \frac{1}{\rho_{p}^{2}} s_{m}\left(\rho_{p}\right) \\
\bar{D}_{q n}^{\alpha \beta} & \equiv \delta_{\alpha \beta}\left[s_{n}^{\prime \prime}\left(\theta_{q}\right)-\left(\frac{l(l+1)}{\cos ^{2} \theta_{q}}+\frac{\lambda(\lambda+1)}{\sin ^{2} \theta_{q}}\right) s_{n}\left(\theta_{q}\right)\right] .
\end{aligned}
$$

Then we use the identity

$$
\bar{A} \otimes \bar{B}+\bar{C} \otimes \bar{D}=\left(\bar{A} U^{-1} \otimes \bar{B} V^{-1}\right)(\Pi \otimes \Xi+I \otimes I)(U \otimes V)
$$

with

$$
\begin{aligned}
& \bar{A}^{-1} \bar{C}=U^{-1} \Pi U \\
& \bar{B}^{-1} \bar{D}=V^{-1} \Xi V
\end{aligned}
$$

and $\Pi, \Xi$ diagonal.

It follows

$$
(\bar{A} \otimes \bar{B}+\bar{C} \otimes \bar{D})^{-1}=\left(U^{-1} \otimes V^{-1}\right)(\Pi \otimes \Xi+I \otimes I)^{-1}\left(U \bar{A}^{-1} \otimes V \bar{B}^{-1}\right) .
$$




\section{TABLES}

\begin{tabular}{|c|c|c|c|c|c|c|}
\hline \multirow[t]{2}{*}{ NN state } & \multirow[t]{2}{*}{$\lambda_{V}$} & \multirow[t]{2}{*}{$r_{\max }$} & \multicolumn{4}{|c|}{$E_{\text {res }}^{2 N}(\mathrm{MeV})$} \\
\hline & & & $\operatorname{exact}(m=l)$ & $\mathrm{m}=0$ & $\mathrm{~m}=1$ & $\mathrm{~m}=2$ \\
\hline${ }^{3} P_{0}$ & 5.50 & $\begin{array}{l}10.0 \\
15.0 \\
20.0\end{array}$ & $\begin{array}{l}1.42-\mathrm{i} 0.75 \\
1.42-\mathrm{i} 0.75 \\
1.42-\mathrm{i} 0.75\end{array}$ & $\begin{array}{l}1.75-\mathrm{i} 1.04 \\
1.58-\mathrm{i} 0.63 \\
1.36-\mathrm{i} 0.58\end{array}$ & & \\
\hline${ }^{1} D_{2}$ & 5.00 & $\begin{array}{l}10.0 \\
15.0 \\
20.0\end{array}$ & $\begin{array}{c}15.08-\mathrm{i} 0.80 \\
15.08-\mathrm{i} 0.80 \\
15.08-\mathrm{i} 0.80\end{array}$ & $\begin{array}{r}15.11-\mathrm{i} 0.90 \\
15.07-\mathrm{i} 0.85 \\
15.06-\mathrm{i} 0.83\end{array}$ & $\begin{array}{r}15.08-\mathrm{i} 0.80 \\
15.08-\mathrm{i} 0.80 \\
15.08-\mathrm{i} 0.80\end{array}$ & \\
\hline${ }^{3} P_{2}-{ }^{3} F_{2}$ & 6.20 & $\begin{array}{l}10.0 \\
15.0 \\
20.0\end{array}$ & $\begin{array}{l}2.79-\mathrm{i} 1.17 \\
2.79-\mathrm{i} 1.17 \\
2.79-\mathrm{i} 1.17\end{array}$ & $\begin{array}{l}3.07-\mathrm{i} 1.05 \\
2.62-\mathrm{i} 0.99 \\
3.08-\mathrm{i} 1.28\end{array}$ & $\begin{array}{l}2.79-\mathrm{i} 1.17 \\
2.79-\mathrm{i} 1.17 \\
2.79-\mathrm{i} 1.17\end{array}$ & \\
\hline
\end{tabular}

TABLE I. Resonance positions in different NN states obtained with the correct (column 4)

and approximate (columns 5-7) boundary conditions applied at different matching radii $r_{\max }$. The Reid93 NN potential multiplied with the factors $\lambda_{V}$ has been used. 


\begin{tabular}{|c|c|c|c|}
\hline $\begin{array}{c}\text { r-matching } \\
(\mathrm{fm})\end{array}$ & $\begin{array}{c}\operatorname{Re}(\alpha) \\
\quad\left({ }^{o}\right)\end{array}$ & $\begin{array}{c}\operatorname{Im}(\alpha) \\
\left({ }^{o}\right)\end{array}$ & $\begin{array}{c}E_{r e s}^{2 N} \\
(\mathrm{MeV})\end{array}$ \\
\hline 10.0 & 0.0 & 0.0 & 1.75 - i 1.04 \\
\hline 10.0 & 20.0 & 0.0 & 1.40 - i 0.88 \\
\hline 10.0 & 0.0 & -40.0 & 1.36 - i 0.58 \\
\hline 10.0 & 0.0 & -80.0 & 1.24 - i 0.58 \\
\hline 10.0 & 20.0 & -40.0 & 1.42 - i 0.73 \\
\hline 10.0 & 20.0 & -80.0 & 1.42 - i 0.75 \\
\hline 2.0 & 20.0 & -80.0 & 1.22 - i 0.93 \\
\hline 2.0 & 20.0 & -160.0 & 1.43 - i 0.75 \\
\hline 20.0 & 20.0 & 0.0 & 1.42 - i 0.73 \\
\hline 20.0 & 30.0 & 0.0 & 1.43 - i 0.74 \\
\hline 20.0 & 40.0 & 0.0 & 1.43 - i 0.75 \\
\hline 20.0 & 44.0 & 0.0 & 1.48 - i 0.75 \\
\hline 20.0 & 46.0 & 0.0 & 1.71 - i 0.72 \\
\hline 20.0 & 48.0 & 0.0 & 2.99 - i 0.36 \\
\hline
\end{tabular}

TABLE II. Complex energy eigenvalues for the ${ }^{3} P_{0} 2 \mathrm{~N}$ state obtained with the Reid $93 \mathrm{NN}$ interaction enhanced by $\lambda_{V}=5.5$ and an approximate boundary condition $(m=0$ term only in Eq.(8) ). The correct energy eigenvalue is $E_{\text {res }}^{2 N}=(1.42-i 0.75) \mathrm{MeV}$. 


\begin{tabular}{|c|c|c|c|c|}
\hline $\begin{array}{c}\text { r-matching } \\
(\mathrm{fm})\end{array}$ & $\begin{array}{c}\lambda \\
\left(f m^{-} 1\right)\end{array}$ & $\begin{array}{c}r_{0} \\
(f m)\end{array}$ & $\begin{array}{c}\alpha \\
\left({ }^{o}\right)\end{array}$ & $\begin{array}{c}E_{r e s}^{2 N} \\
(\mathrm{MeV})\end{array}$ \\
\hline 10.0 & 2.0 & 5.0 & 10.0 & 15.08 - i 0.84 \\
\hline 10.0 & 2.0 & 5.0 & 20.0 & 15.07 - i 0.81 \\
\hline 10.0 & 2.0 & 5.0 & 30.0 & 15.07 - i 0.80 \\
\hline 10.0 & 2.0 & 5.0 & 40.0 & 15.08 - i 0.80 \\
\hline 10.0 & 2.0 & 5.0 & 50.0 & 15.08 - i 0.80 \\
\hline 10.0 & 2.0 & 5.0 & 60.0 & 15.08 - i 0.80 \\
\hline 10.0 & 2.0 & 5.0 & 70.0 & 15.08 - i 0.80 \\
\hline 10.0 & 2.0 & 5.0 & 80.0 & 15.08 - i 0.80 \\
\hline 15.0 & 2.0 & 5.0 & 60.0 & 15.08 - i 0.80 \\
\hline 20.0 & 2.0 & 5.0 & 60.0 & 15.08 - i 0.80 \\
\hline 15.0 & 2.0 & 3.0 & 60.0 & 15.08 - i 0.80 \\
\hline 15.0 & 3.0 & 3.0 & 60.0 & 15.08 - i 0.80 \\
\hline
\end{tabular}

TABLE III. Smooth-exterior complex scaling applied to the ${ }^{1} D_{2}$ resonance state of the Reid93 NN potential with $\lambda_{V}=5.0$. Only the first term in the boundary condition (8) was taken into account. The correct energy eigenvalue is $E_{\text {res }}^{2 N}=(15.08-i 0.80) \mathrm{MeV}$. 


\begin{tabular}{|c|r|r|c|}
\hline \hline NN states & $N_{c}$ & p-space & r-space \\
\hline & & -7.630 & -7.631 \\
\hline${ }^{1} S_{0}+{ }^{3} S_{1}-{ }^{3} D_{1}$ & 5 & -7.403 & -7.406 \\
\hline$j \leq 1$ & 10 & -7.747 & -7.750 \\
\hline$j \leq 2$ & 18 & & \\
\hline \hline
\end{tabular}

TABLE IV. Triton binding energies obtained in momentum- and coordinate-space calculations with the Reid 93 NN potential using different numbers of channels $N_{c}$. 


\begin{tabular}{|c|c|c|c|}
\hline \hline $\begin{array}{c}\rho \text {-matching } \\
(\mathrm{fm})\end{array}$ & $\begin{array}{c}\operatorname{Re}(\alpha) \\
\left({ }^{o}\right)\end{array}$ & $\begin{array}{c}\operatorname{Im}(\alpha) \\
\left(^{o}\right)\end{array}$ & $\begin{array}{c}E_{\text {res }}^{3 B} \\
(\mathrm{MeV})\end{array}$ \\
\hline 10.0 & 0.0 & 0.0 & $8.50-\mathrm{i} 1.81$ \\
10.0 & 20.0 & 0.0 & $8.48-\mathrm{i} 1.87$ \\
10.0 & 40.0 & 0.0 & $8.46-\mathrm{i} 1.86$ \\
5.0 & 20.0 & 0.0 & $8.36-\mathrm{i} 1.78$ \\
5.0 & 40.0 & 0.0 & $8.71-\mathrm{i} 2.03$ \\
5.0 & 40.0 & -20.0 & $8.45-\mathrm{i} 1.93$ \\
5.0 & 40.0 & -40.0 & $8.46-\mathrm{i} 1.86$ \\
5.0 & 40.0 & -60.0 & $8.47-\mathrm{i} 1.86$ \\
\hline \hline
\end{tabular}

TABLE V. CSM applied to a 3-boson system interacting through the S-wave interaction of Eq. (23) in the $J^{\pi}=0^{+}$state. The correct energy eigenvalue is $E_{\text {res }}^{3 B}=(8.47-i 1.86) \mathrm{MeV}$. The boundary condition (21) with A and B terms only has been applied. 


\begin{tabular}{|c|c|c|c|c|}
\hline \hline$\rho_{\text {max }}(\mathrm{fm})$ & \multicolumn{4}{|c|}{$E_{\text {res }}^{3 B}(\mathrm{MeV})$ for particular number of terms in Eq.(21) } \\
\hline & 1 & 2 & 3 & 4 \\
\hline 10.0 & $8.40-\mathrm{i} 1.40$ & $8.50-\mathrm{i} 1.81$ & $8.48-\mathrm{i} 1.85$ & $8.49-\mathrm{i} 1.85$ \\
\hline 15.0 & $8.81-\mathrm{i} 1.80$ & $8.46-\mathrm{i} 1.87$ & $8.46-\mathrm{i} 1.85$ & $8.46-\mathrm{i} 1.85$ \\
\hline 20.0 & $8.00-\mathrm{i} 2.18$ & $8.46-\mathrm{i} 1.85$ & $8.46-\mathrm{i} 1.85$ & $8.46-\mathrm{i} 1.85$ \\
\hline 30.0 & $8.64-\mathrm{i} 1.69$ & $8.47-\mathrm{i} 1.86$ & $8.47-\mathrm{i} 1.86$ & $8.47-\mathrm{i} 1.86$ \\
\hline \hline
\end{tabular}

TABLE VI. Resonance positions of a 3-boson system in the $J^{\pi}=0^{+}$state interacting through the S-wave interaction of Eq.(23). The results are without complex scaling but taking an increasing number of terms in the boundary condition (21) into account. The correct energy eigenvalue is $E_{\text {res }}^{3 B}=(8.47-i 1.86) \mathrm{MeV}$. Number of terms 1 means that only the $A(\theta)$ term has been used. 


\begin{tabular}{|c|r|r|r|r|}
\hline \hline \multicolumn{2}{c}{$\lambda_{V}$} & & $E_{2 n}(\mathrm{MeV})$ & $E_{3 n}(\mathrm{MeV})$ \\
\hline${ }^{1} S_{0}$ & ${ }^{3} P_{0}$ & ${ }^{3} P_{2}-{ }^{3} F_{2}$ & & -31.68 \\
\hline 1.8360 & 9.2804 & 5.2012 & -4.0 & -28.06 \\
1.7241 & 8.9456 & 5.1422 & -3.0 & -24.15 \\
1.5960 & 8.5851 & 5.0797 & -2.0 & -19.73 \\
1.4370 & 8.1846 & 5.0123 & -1.0 & -17.09 \\
1.3297 & 7.9584 & 4.9758 & -0.5 & -14.21 \\
1.1930 & 7.7518 & 4.9442 & -0.1 & -12.96 \\
1.1205 & 7.6981 & 4.9365 & -0.01 & -11.13 \\
1.05 & 7.65 & 4.90 & & -8.24 \\
1.0 & 7.5 & 4.8 & & -7.12 \\
1.0 & 1.0 & 4.8 & & -5.13 \\
1.0 & 1.0 & 4.7 & & -3.29 \\
1.0 & 1.0 & 4.6 & & -1.65 \\
1.0 & 1.0 & 4.5 & & \\
1.0 & 1.0 & & & \\
\hline \hline
\end{tabular}

TABLE VII. Required strength factors $\lambda_{V}$ for particular force components of the Gogny NN interaction together with the corresponding 2- and 3-neutron $\left(3 / 2^{-}\right)$bound energy eigenvalues. 


\begin{tabular}{|c|c|c|c|c|c|c|}
\hline \multirow[t]{2}{*}{$\lambda^{3} P_{2}-{ }^{3} F_{2}$} & \multicolumn{6}{|c|}{$E^{3 n}(\mathrm{MeV})$ for particular number of terms in Eq.(21) } \\
\hline & 1 & 2 & 3 & 4 & 5 & 6 \\
\hline 4.30 & 0.86 - i 0.068 & 0.86 - i 0.068 & 0.85 - i 0.092 & 0.85 - i 0.092 & 0.86 - i 0.068 & 0.86 - i 0.068 \\
\hline 4.25 & 1.26 - i 0.27 & 1.34 - i 0.21 & 1.33 - i 0.21 & 1.34 - i 0.21 & 1.33 - i 0.22 & 1.33 - i 0.22 \\
\hline 4.20 & 1.88 - i 0.38 & 1.77 - i 0.41 & 1.77 - i 0.41 & 1.77 - i 0.41 & 1.79 - i 0.38 & 1.79 - i 0.38 \\
\hline 4.15 & $2.24-\mathrm{i} 0.53$ & 2.20 - i 0.62 & 2.19 - i 0.62 & 2.19 - i 0.63 & $2.21-\mathrm{i} 0.64$ & 2.19 - i 0.63 \\
\hline 4.10 & $2.54-\mathrm{i} 0.70$ & 2.58 - i 0.87 & 2.60 - i 0.86 & $2.58-$ i 0.88 & 2.63 - i 0.89 & 2.57 - i 0.84 \\
\hline 4.05 & $2.72-\mathrm{i} 0.90$ & $2.94-\mathrm{i} 1.15$ & $2.96-\mathrm{i} 1.16$ & 2.97 - i 1.14 & $3.04-\mathrm{i} 1.15$ & $2.92-$ i 1.18 \\
\hline 4.00 & $2.81-\mathrm{i} 1.08$ & 3.27 - i 1.49 & 3.32 - i 1.46 & 3.32 - i 1.46 & $3.31-\mathrm{i} 1.47$ & 3.31 - i 1.47 \\
\hline 3.95 & $2.84-\mathrm{i} 1.20$ & 3.63 - i 1.86 & $3.64-\mathrm{i} 1.81$ & 3.66 - i 1.79 & 3.64 - i 1.80 & 3.64 - i 1.80 \\
\hline 3.90 & $2.84-\mathrm{i} 1.30$ & $4.01-\mathrm{i} 2.24$ & $3.97-$ i 2.16 & $3.95-$ i 2.16 & $3.96-\mathrm{i} 2.14$ & $3.97-$ i 2.14 \\
\hline 3.85 & $2.84-\mathrm{i} 1.37$ & 4.39 - i 2.55 & 4.25 - i 2.53 & 4.24 - i 2.53 & 4.22 - i 2.54 & 4.21 - i 2.51 \\
\hline 3.80 & $2.84-\mathrm{i} 1.41$ & 4.70 - i 2.82 & 4.54 - i 2.91 & 4.50 - i 2.92 & 4.47 - i 2.94 & 4.49 - i 2.93 \\
\hline 3.75 & $2.84-\mathrm{i} 1.44$ & 4.88 - i 3.13 & 4.77 - i 3.29 & 4.76 - i 3.32 & $4.73-$ i 3.33 & 4.78 - i 3.35 \\
\hline 3.70 & $2.84-\mathrm{i} 1.47$ & $5.07-$ i 3.40 & 4.99 - i 3.68 & 4.95 - i 3.75 & 4.98 - i 3.78 & 4.96 - i 3.77 \\
\hline 3.65 & $2.84-\mathrm{i} 1.50$ & $5.17-\mathrm{i} 3.66$ & $5.12-\mathrm{i} 4.08$ & $5.14-\mathrm{i} 4.18$ & $5.16-\mathrm{i} 4.17$ & $5.14-\mathrm{i} 4.19$ \\
\hline 3.60 & $2.84-\mathrm{i} 1.51$ & $5.23-\mathrm{i} 3.91$ & $5.21-\mathrm{i} 4.51$ & $5.30-\mathrm{i} 4.67$ & $5.31-\mathrm{i} 4.68$ & 5.33 - i 4.69 \\
\hline
\end{tabular}

TABLE VIII. The 3-neutron $3 / 2^{-}$resonance state trajectories for the Gogny NN interaction

obtained with increasing number of terms in the boundary condition (21). The enhancement factors $\lambda$ for the ${ }^{1} S_{0}$ and ${ }^{3} P_{0}$ nn force components are equal one. 


\begin{tabular}{|c|c|c|c|c|}
\hline$J^{\pi}$ & \multicolumn{3}{|c|}{$\lambda_{V}$} & $E_{3 n}(\mathrm{MeV})$ \\
\hline & ${ }^{1} S_{0}$ & ${ }^{3} P_{0}$ & ${ }^{3} P_{2}-{ }^{3} F_{2}$ & \\
\hline $1 / 2^{-}$ & 1.0 & 5.5 & 2.75 & 2.82 - i 2.82 \\
\hline $3 / 2^{-}$ & 1.0 & 1.0 & 3.70 & 4.95 - i 3.75 \\
\hline $3 / 2^{+}$ & 1.0 & 5.0 & 4.3 & $5.74-\mathrm{i} 1.53$ \\
\hline
\end{tabular}

TABLE IX. The positions of 3-neutron resonances for the Gogny potential obtained with four leading terms in the boundary condition (21). 


\begin{tabular}{|c|c|c|}
\hline $\begin{array}{c}\operatorname{Re}(\alpha) \\
\left({ }^{o}\right)\end{array}$ & $\begin{array}{c}\operatorname{Im}(\alpha) \\
\left({ }^{o}\right)\end{array}$ & $\begin{array}{c}E_{3 n} \\
(\mathrm{MeV})\end{array}$ \\
\hline 10.0 & 0.0 & 2.88 - i 0.23 \\
\hline 20.0 & 0.0 & 2.88 - i 0.23 \\
\hline 30.0 & 0.0 & 3.11 - i 0.16 \\
\hline
\end{tabular}

TABLE X. CSM applied to the $J^{\pi}=3 / 2^{+}$resonance state of the Gogny potential with enhancement factors $\lambda_{i}=(1.0,5.3,4.6)$. The correct energy eigenvalue is $E_{3 n}^{3 / 2^{+}}=(2.88-i 0.23) \mathrm{MeV}$. 


\begin{tabular}{|r|r|r|r|r|}
\hline \hline$J^{\pi}$ & \multicolumn{5}{|c|}{${ }^{2}$} & $E_{3 n}(\mathrm{MeV})$ \\
\hline & ${ }^{1} S_{0}$ & ${ }^{3} P_{0}$ & ${ }^{3} P_{2}-{ }^{3} F_{2}$ & \\
\hline $3 / 2^{-}$ & 1.0 & 1.0 & 3.25 & $5.30-\mathrm{i} 3.53$ \\
\hline $3 / 2^{+}$ & 1.0 & 4.6 & 3.50 & $5.93-\mathrm{i} 1.55$ \\
\hline \hline
\end{tabular}

TABLE XI. The positions of 3-neutron resonances for the Reid 93 potential obtained with four leading terms in the boundary condition (21). 


\section{FIGURES}

FIG. 1. Analytical continuation of the ${ }^{3} P_{0}$ Reid93 NN potential under the complex scaling transformation $r \rightarrow r e^{i \alpha}$ for five values of $\alpha=0^{\circ}, 20^{\circ}, 40^{\circ}, 60^{\circ}$ and $80^{\circ}$. ((a) real part, (b) imaginary part).

FIG. 2. The real and imaginary parts of the reduced Faddeev amplitudes F for a 3-boson resonance in the state $0^{+}$without complex scaling ((a) real part, (b) imaginary part).

FIG. 3. The same as in Fig.2 but with ECSM taking $\alpha=\left(40.0^{\circ}-i 40.0^{\circ}\right)$ ((a) real part, (b) imaginary part).

FIG. 4. The 3 -neutron $1 / 2^{-}, 3 / 2^{-}$, and $3 / 2^{+}$resonance state trajectories for the Gogny NN interaction obtained with 4 terms in the asymptotic boundary condition. Different points correspond to resonance positions with different values of the enhancement factors. For the $1 / 2^{-}$state $\lambda^{{ } S_{0}}=1.0, \lambda^{3} P_{0}$ changes from 7.5 to 5.5 in steps of 0.1 , and $\lambda^{3} P_{2}-3 F_{2}$ changes from 4.75 to 2.75 in steps of 0.1 . For the $3 / 2^{-}$state $\lambda_{1} S_{0}=1.0, \lambda_{3} P_{0}=1.0$, and $\lambda_{3} P_{2}-3 F_{2}$ changes from 4.3 to 3.7 in steps of 0.05 . For the $3 / 2^{+}$state $\lambda_{S_{0}}=1.0, \lambda_{3} P_{0}$ changes from 5.5 to 5.0 in steps of 0.05 , and ${ }^{\lambda^{3} P_{2}-3 F_{2}}$ changes from 4.8 to 4.3 in steps of 0.05 . 


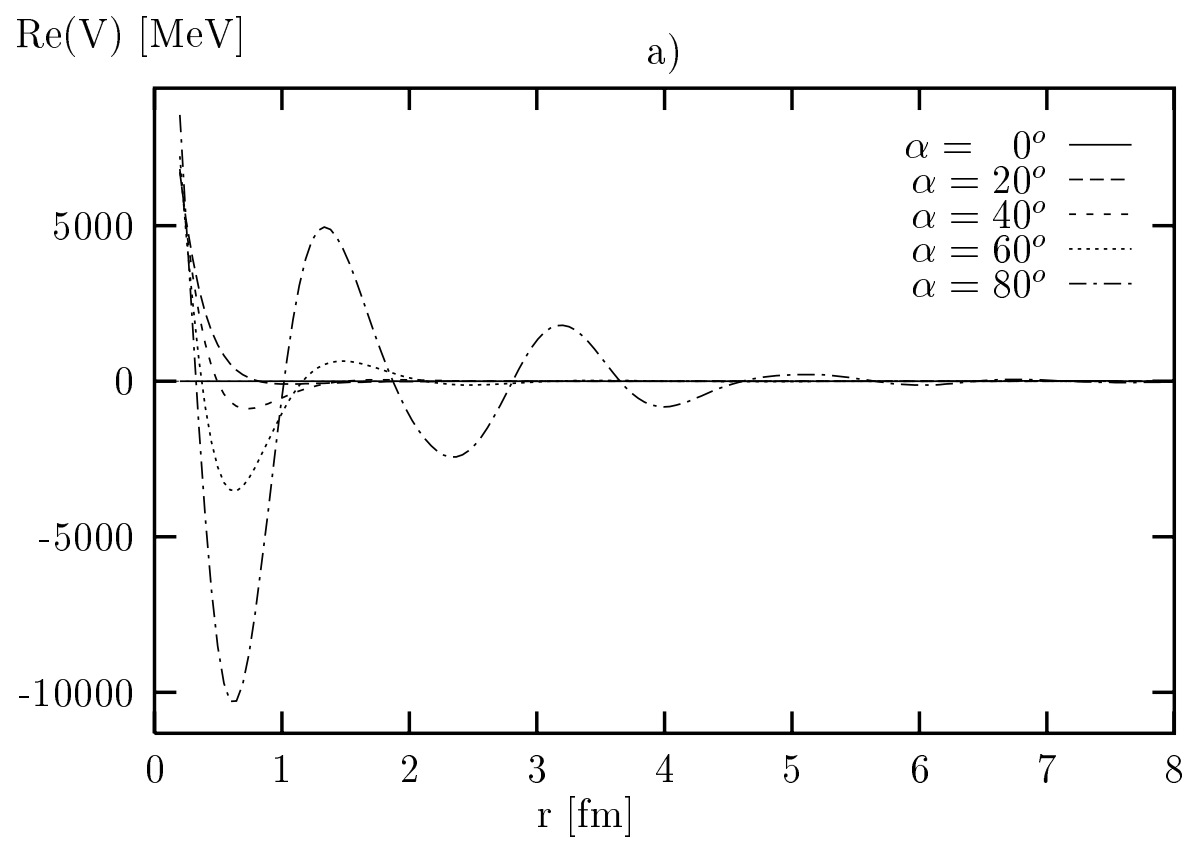




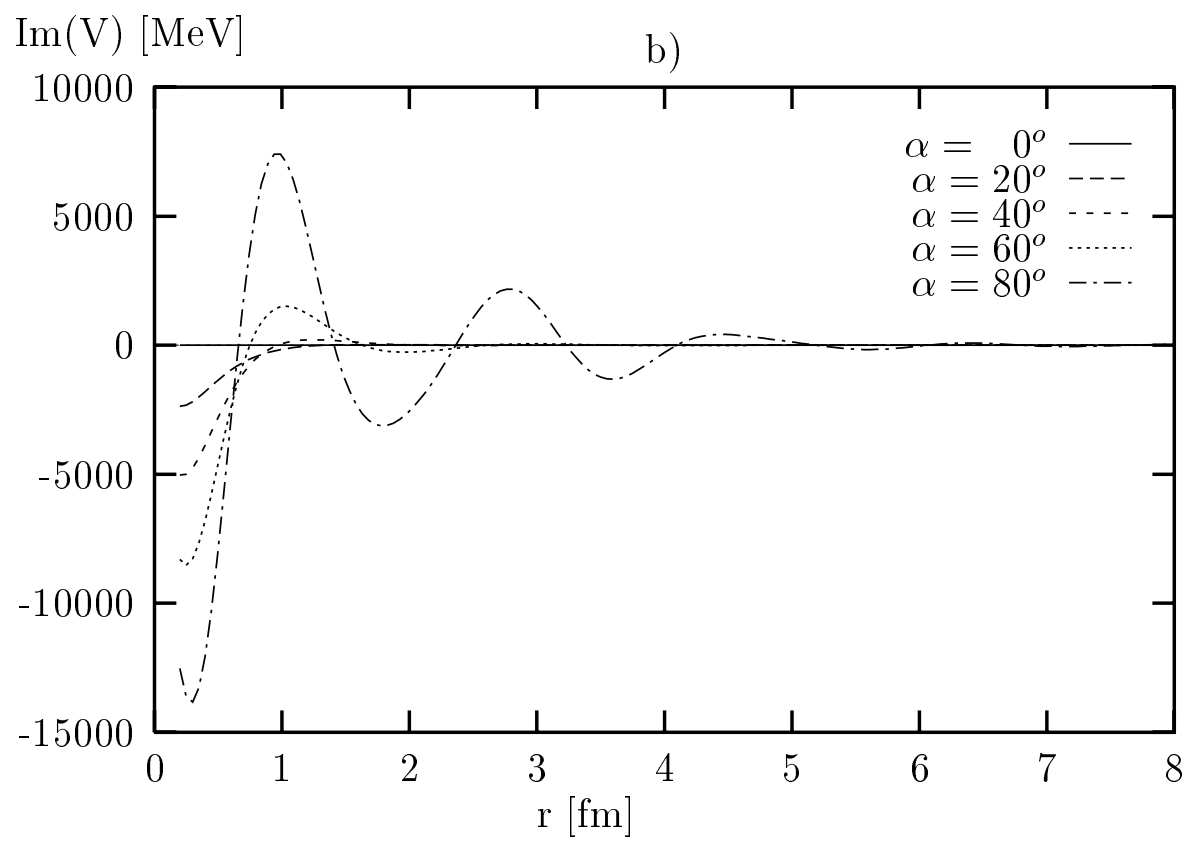


a)

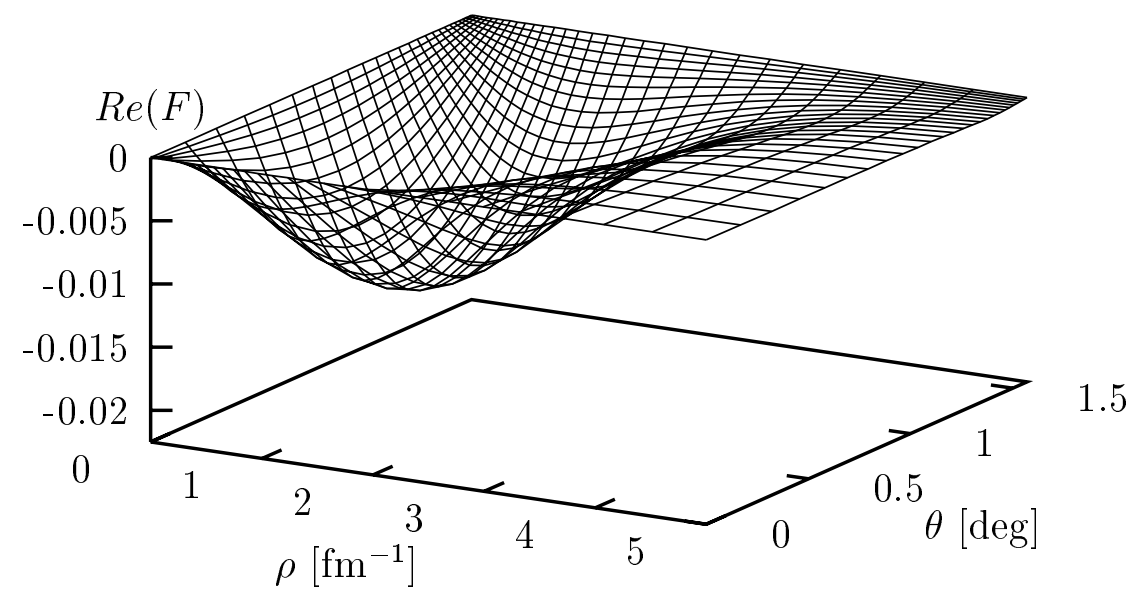




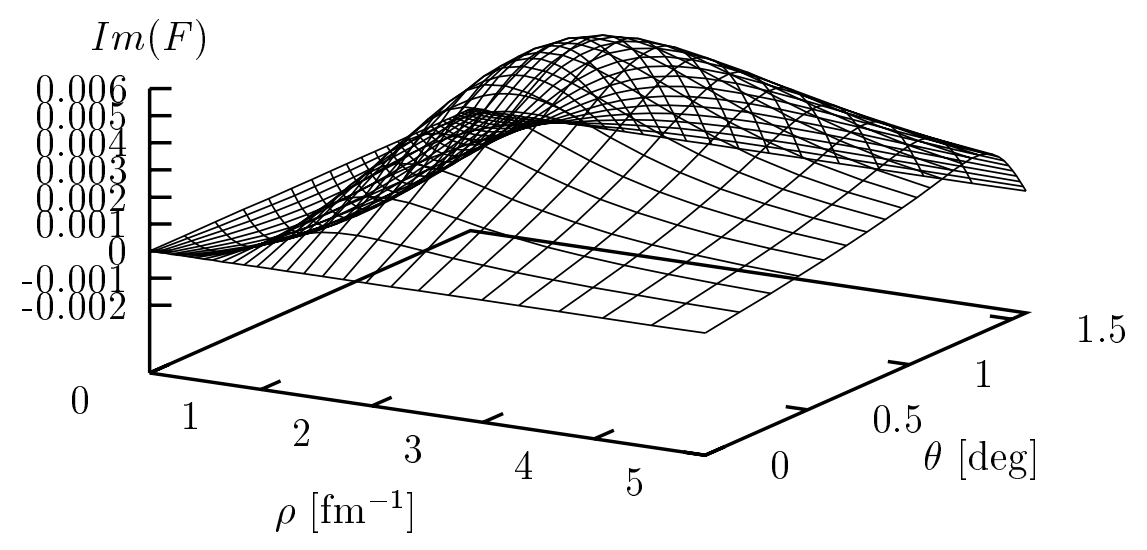




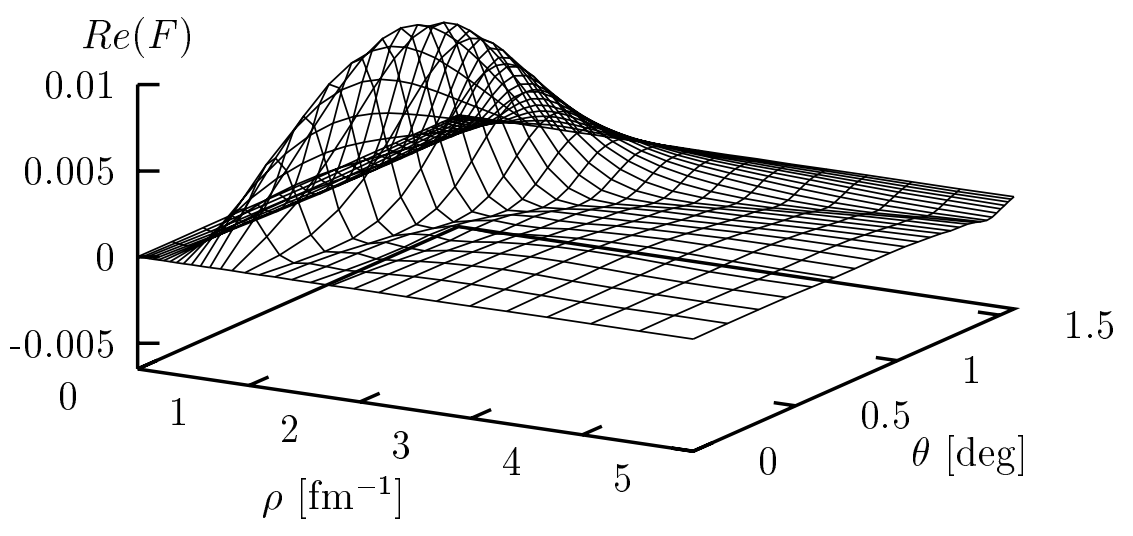


b)

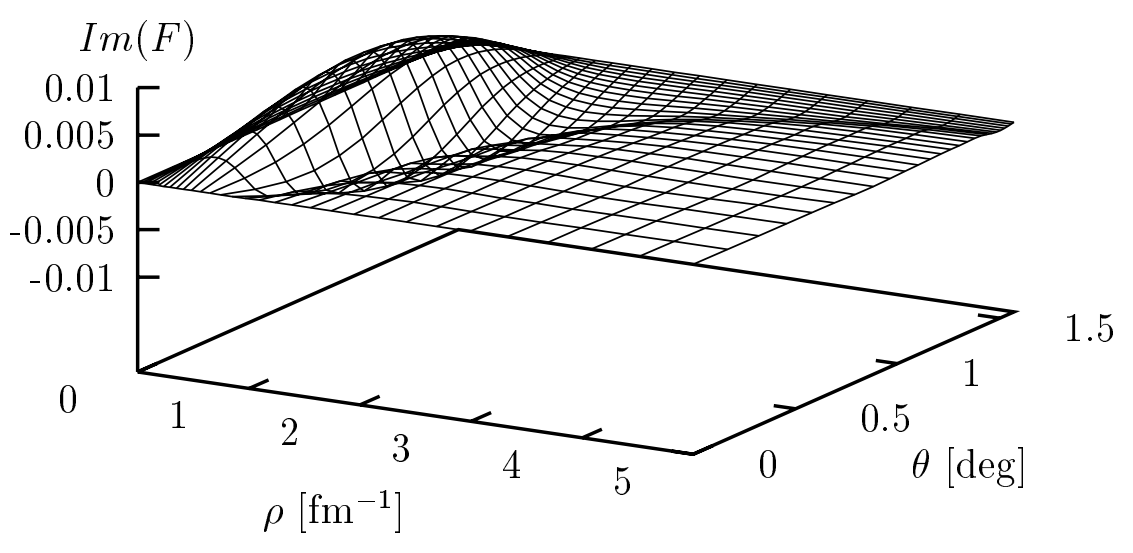




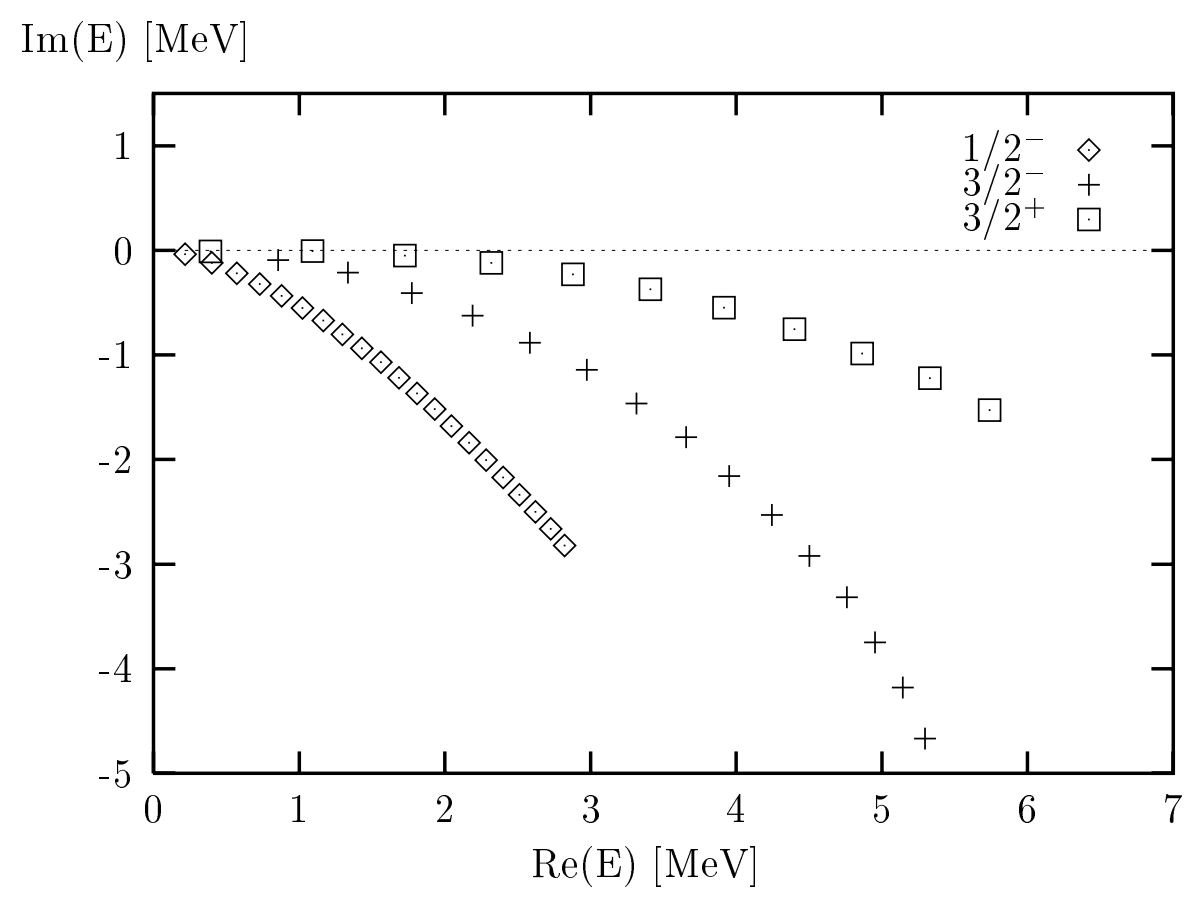

